Homology, Homotopy and Applications, vol. 15(2), 2013, pp.9-51

\title{
HIGHER $K$-THEORY OF KOSZUL CUBES
}

\author{
SATOSHI MOCHIZUKI
}

\author{
(communicated by Charles A. Weibel)
}

\begin{abstract}
The main objective of this paper is to determine generators of the topological filtrations on the higher $K$-theory of a noetherian commutative ring with unit $A$. We introduce the concept of Koszul cubes and give a comparison theorem between the $K$-theory of Koszul cubes with that of topological filtrations.
\end{abstract}

\section{Introduction}

The following generator conjecture is one of the significant problems in commutative algebra and algebraic $K$-theory. (For the relationship between the generator conjecture and Serre's intersection multiplicity conjecture [24], please see the references $[\mathbf{8}],[\mathbf{9}])$.

Let $A$ be a commutative noetherian ring with unit and $p$ a natural number such that $0 \leqslant p \leqslant \operatorname{dim} A$. Let $\mathcal{M}_{A}^{p}$ denote the category of finitely generated $A$-modules $M$ whose support has codimension $\geqslant p$ in $\operatorname{Spec} A$. Recall that a sequence of elements $f_{1}, \cdots, f_{q}$ in $A$ is said to be an $A$-regular sequence if all $f_{i}$ are not unit elements and if $f_{1}$ is not a zero divisor of $A$ and if $f_{i+1}$ is not a zero divisor of $A /\left(f_{1}, \cdots, f_{i}\right)$ for any $1 \leqslant i \leqslant q-1$.

Conjecture 0.1 (Generator conjecture). For any commutative regular local ring $A$ and any natural number $0 \leqslant p \leqslant \operatorname{dim} A$, the Grothendieck group $K_{0}\left(\mathcal{M}_{A}^{p}\right)$ is generated by cyclic modules $A /\left(f_{1}, \cdots, f_{p}\right)$ where the sequence $f_{1}, \cdots, f_{p}$ forms an $A$ regular sequence.

Conjecture 0.1 is equivalent to Gersten's conjecture for $K_{0}$. Here is a statement of Gersten's conjecture for $K_{n}$ :

For any commutative regular local ring $A$ and natural numbers $n, p$, the canonical inclusion $\mathcal{M}_{A}^{p+1} \hookrightarrow \mathcal{M}_{A}^{p}$ induces the zero map on $K$-groups

$$
K_{n}\left(\mathcal{M}_{A}^{p+1}\right) \rightarrow K_{n}\left(\mathcal{M}_{A}^{p}\right)
$$

where $K_{n}\left(\mathcal{M}_{A}^{i}\right)$ denotes the $n$-th $K$-group of the abelian category $\mathcal{M}_{A}^{i}$. (See $\left.[\mathbf{1 0}]\right)$.

Remark. Conjecture 0.1 is known for the following cases:

Received October 18, 2012, revised November 29, 2012, March 16, 2013; published on August 19, 2013.

2010 Mathematics Subject Classification: 13D02, 14C35, 19D50.

Key words and phrases: higher $K$-theory, Koszul cube.

Article available at http://intlpress.com/HHA/v15/n2/a2 and doi:10.4310/HHA.2013.v15.n2.a2

Copyright (C) 2013, International Press. Permission to copy for private use granted. 
(i) $A=B\left[\left[T_{1}, \cdots, T_{n}\right]\right] /\left(\Sigma T_{i}^{2}-\pi\right)$, where $B$ is a discrete valuation ring and $\pi$ is a prime element in $B$ or an unramified regular local ring $A$ by combining the result in $[\mathbf{2 2}],[\mathbf{2 1}],[\mathbf{1 1}]$ and $[\mathbf{1 7}]$. (Please see also related works $[\mathbf{2}]$ and $[\mathbf{5}]$ ).

(ii) $p=0,1,2$ and $\operatorname{dim} A$ (The results are classical for $p=0$ and $p=\operatorname{dim} A$ and for $p=1$ and $p=2$, please see the reference $[\mathbf{2 5}]$ ).

Let $\mathcal{M}_{A}^{p}(p)$ denote the full subcategory of $\mathcal{M}_{A}^{p}$ consisting of those $A$-modules $M$ of projective dimension $\leqslant p$. It is well-known that if $A$ is regular, then the canonical inclusion functor $\mathcal{M}_{A}^{p}(p) \hookrightarrow \mathcal{M}_{A}^{p}$ induces a homotopy equivalence on $K$-theory. (See 5.11). For any endomorphism of $A$-module $\phi: F \rightarrow F$ between a finitely generated free $A$-module $F$, if we fix a basis $\alpha$ of $F$, then $\phi$ is represented by a square matrix $\Phi$. We write $\operatorname{det}_{\alpha} \phi$ or simply $\operatorname{det} \phi$ for $\operatorname{det} \Phi$ and call it the determinant of $\phi$ (with respect to $\alpha$ ). In connection with Conjecture 0.1 , here is a corollary to the main theorem in this paper.

Theorem 0.2. If $A$ is a local Cohen-Macaulay ring, then for any natural number $0 \leqslant p \leqslant \operatorname{dim} A$, the Grothendieck group $K_{0}\left(\mathcal{M}_{A}^{p}(p)\right)$ is generated by modules of the form

$$
F /\left\langle\operatorname{Im} \psi_{1}, \cdots, \operatorname{Im} \psi_{p}\right\rangle,
$$

where $F$ is a finitely generated free $A$-module and $\psi_{k}: F \rightarrow F$ is an A-module homomorphism such that the sequence $\operatorname{det} \psi_{1}, \cdots, \operatorname{det} \psi_{p}$ forms an $A$-regular sequence for any basis of $F$.

Remark. It is well-known that in general $K_{0}\left(\mathcal{M}_{A}^{3}(3)\right)$ is not generated by cyclic modules $A /\left(f_{1}, f_{2}, f_{3}\right)$, where $f_{1}, f_{2}, f_{3}$ forms a regular sequence. Please see the reference [7], [18] and [1]. On the other hand, Smoke proved that for any $\operatorname{dim} A \geqslant p \geqslant 3$, $K_{0}\left(\mathcal{M}_{A}^{p}(p)\right)$ is generated by cyclic modules $A /\left(f_{1}, \cdots, f_{r}\right)(r \geqslant p)$, where the sequence $f_{1}, \cdots, f_{r}$ forms an $A$-regular sequence. Please see the reference $[\mathbf{2 5}, 4.2]$.

More generally, the main objective in the paper is to study topological filtrations on the higher $K$-theory of a commutative noetherian ring with unit. Inspired by the works of Gillet and Soulé [12], of Diekert [6], and of Grayson [14], the main method in the paper is to replace certain full subcategories of modules with the category of cubes in the category of appropriate modules. More accurately, let us fix a commutative noetherian ring with unit $A$, a non-negative integer $q$ and a sequence $f_{1}, \ldots, f_{p}$ in $A$ such that for any bijection $\sigma$ on the set $S=\{1, \ldots, p\}, f_{\sigma(1)}, \ldots, f_{\sigma(p)}$ is an $A$-regular sequence. We put $I=\left(f_{1}, \ldots, f_{p}\right)$ and $\mathfrak{f}_{S}=\left\{f_{s}\right\}_{s \in S}$. Let $\mathcal{M}_{A}^{I}(q)$ denote the category of finitely generated $A$-modules $M$ such that $\operatorname{Projdim}_{A} M \leqslant q$ and Supp $M \subset V(I)$. (See Notations 4.6).

A Koszul cube $x$ associated with $f_{1}, \ldots, f_{p}$ is a contravariant functor from $[1]^{\times p}$ to the category of finitely generated projective $A$-modules $\mathcal{P}_{A}$, where [1] is the totally ordered set $\{0,1\}$ with the natural order $0<1$ satisfying the condition that for each $1 \leqslant k \leqslant p$ and $\mathfrak{i}=\left(i_{1}, \ldots, i_{p}\right) \in[1]^{\times p}$ such that $i_{k}=1, d_{\mathfrak{i}}^{k}:=x\left(\mathfrak{i}-\mathfrak{e}_{k} \rightarrow \mathfrak{i}\right)$ is injective and Coker $d_{\mathfrak{i}}^{k}$ is in $\mathcal{M}_{A}^{f_{k} A}(1)$, where $\mathfrak{e}_{k}$ is the $k$-th unit vector. A morphism between Koszul cubes is just a natural transformation. We write $\operatorname{Kos}_{A}^{\mathfrak{f}_{S}}$ for the category of Koszul cubes associated with $f_{1}, \ldots, f_{p}$. (See Definition 4.8). A Koszul cube $x$ associated with $f_{1}, \ldots, f_{p}$ is reduced if for each $1 \leqslant k \leqslant p$ and $\mathfrak{i}=\left(i_{1}, \ldots, i_{p}\right) \in[1]^{\times p}$ such 
that $i_{k}=1, f_{k}$ Coker $d_{\mathfrak{i}}^{k}=0$. We write $\operatorname{Kos}_{A \text {,red }}^{\mathfrak{f}_{S}}$ for the category of reduced Koszul cubes associated with the family $\mathfrak{f}_{S}=\left\{f_{s}\right\}_{s \in S}$. (See Lemma-Definition 5.4). If we consider a Koszul cube $x$ as a multi-complex, where $x_{(0, \ldots, 0)}$ is in degree $(0, \ldots, 0)$, we will take its total complex. (See Definition 3.5). We will prove that for any Koszul cube $x, \mathrm{H}_{k}(\operatorname{Tot} x)=0$ for $k>0$ (See 3.15, 4.19). A morphism between Koszul cubes $f: x \rightarrow y$ is a quasi-isomorphism if $\mathrm{H}_{0}$ Tot $f$ is an isomorphism. We denote the class of quasi-isomorphisms in $\operatorname{Kos}_{A}^{\mathfrak{f}_{S}}$ and $\mathbf{K o s}_{A, \text { red }}^{\mathfrak{f}_{S}}$ by the same symbol tq. The term "Koszul" comes from the fact that the total complex of the cube of Example 2.7 is just the usual Koszul complex associated with $\left\{f_{s}\right\}_{s \in S}$. We have the morphism of Waldhausen categories

$$
\mathrm{H}_{0} \text { Tot: }\left(\operatorname{Kos}_{A}^{\mathfrak{f}_{S}}, \mathrm{tq}\right) \rightarrow\left(\mathcal{M}_{A}^{\mathfrak{f}_{S}}(p), i\right),
$$

where $i$ is the class of all isomorphisms. The next result is the comparison theorem referred to in the Abstract.

Theorem 0.3 (A part of Corollary 5.14). The exact functor $\mathrm{H}_{0}$ Tot: $\mathbf{K o s}_{A}^{\mathfrak{f}_{S}} \rightarrow$ $\mathcal{M}_{A}^{\mathfrak{f}_{S}}(p)$ induces a homotopy equivalence on $K$-theory:

$$
K\left(\operatorname{Kos}_{A}^{\mathfrak{f}_{S}} ; \mathrm{tq}\right) \rightarrow K\left(\mathcal{M}_{A}^{\mathfrak{f}_{S}}(p)\right) .
$$

When $A$ is a principle ideal domain, Theorem 0.3 has been proven in $[\mathbf{2 0}]$. To prove the theorem above, we develop a resolution theorem for Waldhausen categories. (See Theorem 1.13). The other ingredient of the proof is giving a quite elementary, but new algorithm of resolution process of modules by finite direct sums of typical Koszul cubes. (See Theorem 5.12). The second main theorem is the following:

Theorem 0.4 (See Corollary 6.3). In the notation above, moreover if we assume that $A$ is regular, then the canonical inclusion functor $\iota: \mathbf{K o s}_{A, \text { red }}^{\mathfrak{f}_{S}} \hookrightarrow \mathbf{K o s}_{A}^{\mathfrak{f}_{S}}$ induces the following homotopy equivalences on K-theory:

$$
\begin{aligned}
K\left(\mathbf{K o s}_{A, \text { red }}^{\mathfrak{f}_{S}}\right) & \rightarrow K\left(\mathbf{K o s}_{A}^{\mathfrak{f}_{S}}\right) \\
K\left(\operatorname{Kos}_{A, \text { red }}^{\mathfrak{f}_{S}} ; \mathrm{tq}\right) & \rightarrow K\left(\operatorname{Kos}_{A}^{\mathfrak{f}_{S}} ; \text { tq }\right) .
\end{aligned}
$$

To prove the theorem above, we will utilize the split fibration theorem 2.19 which is a generalization of Lemma 3.3 in [20]. Theorem 0.4 has the following application to Gersten's conjecture:

Corollary 0.5. Gersten's conjecture for a regular local ring $A$ is equivalent to the following assertion: For any A-regular sequence $\left\{f_{s}\right\}_{s \in S}$ in $A, \mathrm{H}_{0}$ Tot: $\operatorname{Kos}_{\mathrm{red}, A}^{\mathfrak{f}_{S}} \rightarrow$ $\mathcal{M}_{A}^{\# S-1}$ induces the zero maps on $K$-groups.

The relationship between Gersten's conjecture and weight of the Adams operations on Koszul cubes, a higher analogue of generator conjecture will be studied in my subsequent papers by utilizing Corollary 0.5.

By handling koszul cubes, in particular free Koszul cubes, we will be able to import linear algebra and combinatorial methods into our research for modules and (perfect) complexes in my forthcoming papers. On the other hand, since Waldhausen categories of Koszul cubes are not closed under taking the mapping cylinder functor, many standard theorems in Waldhausen $K$-theory, such as the generic fibration theorem 
and the approximation theorem in the literature do not apply directly. The paper is devoted to the concept of Koszul cubes, for example, homological algebra for cubes, and the fundamental technique of manipulating $K$-theory for Waldhausen category without assuming the factorization axiom.

Now we give a guide for the structure of this paper. In section 1, we give a resolution theorem for Waldhausen categories which is a generalization of Quillen's original one in $[\mathbf{2 2}]$. In section 2 , we develop the theory of semi-direct products of exact categories which is initiated in [20]. In section 3, we establish the theory about admissible cubes in an abelian category which is a categorical variant of the concept about regular sequences. We will calculate homology groups of the total complex associated with an admissible cube and utilizing this, we give several characterizations of admissibility. Finally we extend the notion of semi-direct products to that of multi semi-direct products of a family of exact categories. In section 4, we define Koszul cubes and by combining results in the previous sections, we describe the category of Koszul cubes by multi semi-direct products of the exact categories of pure weight modules. In section 5, we give the algorithm of resolution process as mentioned above and as its corollary we get the first main result. In the final section, assuming the regularity of $A$, we will prove a dévissage theorem for Koszul cubes.

\section{Conventions.}

\section{(1) Set theory}

(i) Throughout this paper, we use the letter $S$ to denote a set.

(ii) For a positive integer $n$, we write $(n]$ for the set of integers $k$ such that $1 \leqslant k \leqslant n$ and for a non-negative integer $m$, we denote the totally ordered set of integers $k$ such that $0 \leqslant k \leqslant m$ by $[m]$.

(iii) For any set $S$, we write $\mathcal{P}(S)$ for its power set. Namely $\mathcal{P}(S)$ is the set of all subsets of $S$. We consider $\mathcal{P}(S)$ to be a partially ordered set under inclusion. A fortiori, $\mathcal{P}(S)$ is a category.

(iv) For a finite set $S$, we denote the number of elements in $S$ by $\# S$.

\section{(2) Commutative algebra}

(i) Throughout this paper, we use the letter $R$ (resp., $A$ ) to denote a commutative ring with 1 (resp., commutative noetherian ring with 1 ).

(ii) For any $R$, we write $R^{\times}$for the group of units in $R$.

(iii) If $\left\{f_{s}\right\}_{s \in S}$ is a subset of $R$, we write $\mathfrak{f}_{S}$ for the ideal they generate. By convention, we set $\mathfrak{f}_{\emptyset}=(0)$.

(iv) A subring of $R$ is a subring with the same $1=1_{R}$.

(v) For any $R, A$, we let $\mathcal{P}_{R}$ denote the category of finitely generated projective $R$-modules, and let $\mathcal{M}_{A}$ denote the category of finitely generated $A$-modules.

\section{(3) Category theory}

(i) Throughout the paper, we use the letters $\mathcal{C}$ and $\mathcal{A}$ to denote a category and an abelian category, respectively.

(ii) For any category $\mathcal{C}$, we denote the class of objects in $\mathcal{C}$ by $\mathrm{Ob} \mathcal{C}$.

(iii) For any category $\mathcal{C}, i \mathcal{C}$ or just $i$ means the subcategory of all isomorphisms in $\mathcal{C}$. 
(iv) For categories $\mathcal{X}$ and $\mathcal{Y}$, let us denote the (large) category of functors from $\mathcal{X}$ to $\mathcal{Y}$ by $\mathcal{H O M}(\mathcal{X}, \mathcal{Y})$.

(v) For categories $\mathcal{X}$ and $\mathcal{Y}$, a functor from $\mathcal{X}$ to $\mathcal{Y}, j: \mathcal{X} \rightarrow \mathcal{Y}$ is an inclusion functor if it is fully faithful and the function between their classes of objects $j: \mathrm{Ob} \mathcal{X} \rightarrow \mathrm{Ob} \mathcal{Y}$ is injective. We denote an inclusion functor by the arrow "ฯ".

\section{(4) Exact categories, Waldhausen categories and algebraic $K$-theory}

(i) Basically, for exact categories, we follow the notations in $[\mathbf{2 2}]$ and for algebraic $K$-theory of categories with cofibrations and weak equivalences, we follow the notations in [29].

(ii) We denote an admissible monomorphism (resp. an admissible epimorphism) by the arrow “ $\longmapsto$ " (resp. “ $\rightarrow ")$.

(iii) We call a category with cofibrations and weak equivalences a Waldhausen category.

(iv) For a Waldhausen category $(\mathcal{X}, w)$, we denote its $\mathcal{S}$-construction by $w \mathcal{S}_{\bullet} \mathcal{X}$ and write $K(\mathcal{X} ; w)$ for the $K$-space $\Omega\left|w \mathcal{S}_{\bullet} \mathcal{X}\right|$. We also write $K(\mathcal{X})$ for $K(\mathcal{X} ; i)$.

(v) We say that a functor between exact categories (resp. categories with cofibrations) $f: \mathcal{X} \rightarrow \mathcal{Y}$ reflects exactness if for a sequence $x \rightarrow y \rightarrow z$ in $\mathcal{X}$ such that $f x \rightarrow f y \rightarrow f z$ is an admissible exact sequence (resp. a cofibration sequence) in $\mathcal{Y}, x \rightarrow y \rightarrow z$ is an admissible exact sequence (resp. a cofibration sequence) in $\mathcal{X}$.

(vi) For an exact category $\mathcal{E}$, we say that its full subcategory $\mathcal{F}$ is an exact subcategory (resp. a strict exact subcategory) if it is an exact category and the inclusion functor is exact (and reflects exactness).

(vii) Notice that as in $[\mathbf{2 9}$, p.321, p.327], the concept of subcategories with cofibrations (resp. Waldhausen subcategories) is stronger than that of exact subcategories. Namely we say that $\mathcal{C}^{\prime}$ is a subcategory with cofibrations of a category with cofibration $\mathcal{C}$ if a morphism in $\mathcal{C}^{\prime}$ is a cofibration in $\mathcal{C}^{\prime}$ if and only if it is a cofibration in $\mathcal{C}$ and the quotient is in $\mathcal{C}^{\prime}$ (up to isomorphism). That is, the inclusion functor $\mathcal{C}^{\prime} \hookrightarrow \mathcal{C}$ is exact and reflects exactness. For example, let $\mathcal{E}$ be a non-semisimple exact category. Then $\mathcal{E}$ with semi-simple exact structure is not a subcategory with cofibrations of $\mathcal{E}$, but a exact subcategory of $\mathcal{E}$.

(viii) Let $\mathcal{E}$ be an exact category and $\mathcal{F}$ a full subcategory of $\mathcal{E}$. We say that $\mathcal{F}$ is closed under kernels (of admissible epimorphisms) if for any admissible exact sequence $x \longmapsto y \rightarrow z$ in $\mathcal{E}$ if $y$ is isomorphic to object in $\mathcal{F}$, then $x$ is also isomorphic to an object in $\mathcal{F}$. (See [31, II.7.0]).

(ix) We say that the class of morphisms $w$ in an exact category $\mathcal{E}$ satisfies the cogluing axiom if $\left(\mathcal{E}^{o p}, w^{o p}\right)$ satisfies the gluing axiom.

(x) A pair of an exact category $\mathcal{E}$ and a class of morphisms $w$ in $\mathcal{E}$ is said to be a Waldhausen exact category if $(\mathcal{E}, w)$ and $\left(\mathcal{E}^{o p}, w^{o p}\right)$ are Waldhausen categories.

(xi) For a Waldhausen category $(\mathcal{C}, w)$, we write $w(\mathcal{C})$ if we wish to emphasis that $w$ is the class of weak equivalences in $\mathcal{C}$. We write $\mathcal{C}$ for $(\mathcal{C}, w)$ when $w$ is the class of all isomorphisms in $\mathcal{C}$. 
(xii) An object $x$ in a Waldhausen category $(\mathcal{C}, w)$ is $w$-trivial if the canonical morphism $0 \rightarrow x$ is in $w$. We write $\mathcal{C}^{w}$ for the full subcategory of $w$-trivial objects of $\mathcal{C}$.

(xiii) For a Waldhausen category $\mathcal{C}$ and subcategories with cofibrations $\mathcal{X}$ and $\mathcal{Y}$ of $\mathcal{C}$, let $E(\mathcal{X}, \mathcal{C}, \mathcal{Y})$ denote the category with cofibrations of cofibration sequences $x \longmapsto y \rightarrow z$ in $\mathcal{C}$ such that $x$ is in $\mathcal{X}$ and $z$ is in $\mathcal{Y}$.

\section{A resolution theorem for Waldhausen categories}

In this section, we will prove a resolution theorem for Waldhausen categories by improving the proof for exact categories in $[\mathbf{2 6}]$. This theorem is a generalization of Quillen's original one in $[\mathbf{2 2}]$. Let $(\mathcal{X}, w)$ be a Waldhausen category and $\mathcal{Y}$ a full subcategory of $\mathcal{X}$ closed under extensions in $\mathcal{X}$. In 1.9 and 1.12 , we will define the (strong) resolution conditions of the inclusion functor $\iota: \mathcal{Y} \hookrightarrow \mathcal{X}$. In this situation, $\mathcal{Y}$ naturally becomes a Waldhausen subcategory and if $\mathcal{X}$ is essentially small, then the canonical map induced by $\iota, K(\mathcal{Y} ; w) \rightarrow K(\mathcal{X} ; w)$ is a homotopy equivalence 1.13 . In this section, from now on, let $(\mathcal{X}, w)$ be a Waldhausen category.

Definition 1.1. Let $v$ be a class of morphisms in a category $\mathcal{C}$. We say that $v$ is a multiplicative system of $\mathcal{C}$ if $v$ is closed under finite compositions and closed under isomorphisms. Namely

(1) if $\bullet \stackrel{f}{\rightarrow} \bullet \stackrel{g}{\rightarrow} \bullet$ are composable morphisms in $v$, then $g f$ is also in $v$, and

(2) all isomorphisms in $\mathcal{C}$ are in $v$.

For a category $\mathcal{C}$ and a multiplicative system $v$ of $\mathcal{C}$, we define the simplicial subcategory $\mathcal{C}(-, v)$ in $\mathcal{H O M}(-, \mathcal{C})$

$$
[m] \mapsto \mathcal{C}(m, v),
$$

where $\mathcal{C}(m, v)$ is the full subcategory of $\mathcal{H O M}([m], \mathcal{C})$ consisting of those functors which take values in $v$. For each $m$, we denote an object $x \bullet$ in $\mathcal{C}(m, v)$ by

$$
x_{\bullet}: x_{0} \stackrel{i_{0}^{x}}{\rightarrow} x_{1} \stackrel{i_{1}^{x}}{\rightarrow} x_{2} \stackrel{i_{2}^{x}}{\rightarrow} \cdots \stackrel{i_{m-1}^{x}}{\rightarrow} x_{m} .
$$

Example 1.2. (cf. $[\mathbf{2 9}, 1.1 .4]$.$) . For a category with cofibrations (\mathcal{Z}$, Cof $\mathcal{Z})$ and each non-negative integer $m$, we can naturally make $F_{m} \mathcal{Z}:=\mathcal{Z}(m$, Cof $\mathcal{Z})$ into a category with cofibrations. Here a morphism $a_{\bullet} \rightarrow a_{\bullet}^{\prime}$ is defined to be a cofibration if for each $0 \leqslant j \leqslant m, a_{j} \rightarrow a_{j}^{\prime}$ and $a_{j}^{\prime} \sqcup_{a_{j}} a_{j+1} \rightarrow a_{j+1}^{\prime}$ are cofibrations in $\mathcal{Z}$.

Example 1.3. (cf. [29, p.336 in the proof of 1.4.3.]). For $(\mathcal{X}, w)$ and each non-negative integer $m$, we can make $\mathcal{X}(m, w)$ into a category with cofibrations by defining the cofibrations to be term-wised cofibrations in $\mathcal{X}$.

Lemma 1.4. Let $\mathcal{D}$ be a full subcategory of a category $\mathcal{C}, v$ a multiplicative system in $\mathcal{C}$ and $m$ a non-negative integer. For each $x_{\bullet}$ in $\mathcal{C}(m, v)$ if each $x_{i}$ is isomorphic to an object in $\mathcal{D}$, then $x$. is isomorphic to an object in $\mathcal{D}(m, v)$.

Proof. For each $x_{j}$, there are an object $y_{j}$ in $\mathcal{Y}$ and an isomorphism $\phi_{j}: x_{j} \stackrel{\sim}{\rightarrow} y_{j}$. We define the morphism $i_{j}^{y}: y_{j} \rightarrow y_{j+1}$ by the formula $i_{j}^{y}:=\phi_{j+1} i_{j}^{x} \phi_{j}^{-1}$. Since $v$ is a 
multiplicative system, $i_{j}^{y}$ is in $v$ and therefore

$$
y_{\bullet}: y_{0} \stackrel{i_{a}^{y}}{\rightarrow} y_{1} \stackrel{i_{y}^{y}}{\rightarrow} y_{2} \stackrel{i_{2}^{y}}{\rightarrow} \ldots \stackrel{i_{m-1}^{y}}{\rightarrow} y_{m}
$$

is an object in $\mathcal{D}(m, v)$ and it is isomorphic to $x_{\bullet}$.

Definition 1.5. Let $\mathcal{Y}$ be a full subcategory of $\mathcal{X} . \mathcal{Y}$ is said to be closed under extensions in $\mathcal{X}$ if for a cofibration sequence $x \longmapsto y \rightarrow z$ in $\mathcal{X}, x$ and $z$ are isomorphic to objects in $\mathcal{Y}$, respectively, then $y$ is also isomorphic to an object in $\mathcal{Y}$. In this case, $\mathcal{Y}$ is a Waldhausen category by declaring that a morphism $x \rightarrow y$ in $\mathcal{Y}$ is a cofibration in $\mathcal{Y}$ if it is a cofibration in $\mathcal{X}$ and if $y / x$ is isomorphic to an object in $\mathcal{Y}$ and that a morphism $x \rightarrow y$ in $\mathcal{Y}$ is a weak equivalence in $\mathcal{Y}$ if it is a weak equivalence in $\mathcal{X}$. From now on, let $\mathcal{Y}$ be a full subcategory of $\mathcal{X}$ closed under extensions.

Remark 1.6. The extensional closed condition is preserved by equivalences as categories with cofibrations. That is, let us consider the commutative diagram of categories with cofibrations

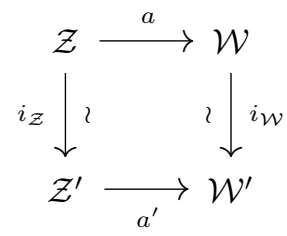

with both $i_{\mathcal{Z}}$ and $i_{\mathcal{W}}$ are fully faithful, essentially surjective and exact and reflect exactness. If $a: \mathcal{Z} \hookrightarrow \mathcal{W}$ is closed under extensions in $\mathcal{W}$, then $a^{\prime}: \mathcal{Z}^{\prime} \hookrightarrow \mathcal{W}^{\prime}$ is also closed under extensions in $\mathcal{W}^{\prime}$.

Lemma 1.7. In the case above, for any non-negative integer $m$, the inclusion functors

$$
\begin{aligned}
\mathcal{Y}(m, w) & \hookrightarrow \mathcal{X}(m, w), \\
F_{m} \mathcal{Y} & \hookrightarrow F_{m} \mathcal{X} \text { and } \\
\mathcal{S}_{m} \mathcal{Y} & \hookrightarrow \mathcal{S}_{m} \mathcal{X}
\end{aligned}
$$

are closed under extensions in $\mathcal{X}(m, w), F_{m} \mathcal{X}$ and $\mathcal{S}_{m} \mathcal{X}$, respectively.

Proof. Let us consider a cofibration sequence

$$
x_{\bullet} \longmapsto y_{\bullet} \rightarrow z_{\bullet}
$$

in $\mathcal{X}(m, w)$ or $F_{m} \mathcal{X}$ and assume that $x_{\bullet}$ and $z_{\bullet}$ are isomorphic to objects in $\mathcal{Y}(m, v)$ or $F_{m} \mathcal{Y}$, respectively. Then by the definitions (see 1.2 or 1.3 ), for each $0 \leqslant j \leqslant m$, we have the cofibration sequence

$$
x_{j} \longmapsto y_{j} \rightarrow z_{j}
$$

in $\mathcal{X}$. Therefore by assumption, $y_{j}$ is isomorphic to an object in $\mathcal{Y}$. Now by 1.4 , we learn that $y_{\bullet}$ is isomorphic to an object in $\mathcal{Y}(m, v)$ or $F_{m} \mathcal{Y}$. This means that $\mathcal{Y}(m, w)$, $F_{m} \mathcal{Y}$ are closed under extensions in $\mathcal{X}(m, v)$ or $F_{m} \mathcal{X}$, respectively. Finally since we have the functorial equivalence $F_{m-1} \mathcal{X} \stackrel{\sim}{\rightarrow} \mathcal{S}_{m} \mathcal{X}$ as categories with cofibrations, we notice that $\mathcal{S}_{m} \mathcal{Y}$ is closed under extensions in $\mathcal{S}_{m} \mathcal{X}$ by 1.6. 
Definition 1.8. In the situation above, we can define the category $\mathcal{X}_{\mathcal{Y}}$ as follows. The class of objects of $\mathcal{X}_{\mathcal{Y}}$ is same as that of $\mathcal{X}$. A morphism $x \rightarrow y$ in $\mathcal{X}$ is a cofibration in $\mathcal{X}$ such that $y / x$ is isomorphic to an object in $\mathcal{Y}$. One can easily prove that morphisms in $\mathcal{X}$ y are closed under compositions, namely it is actually a category by virtue of the assumption 1.5. Notice that there is the natural inclusion functor $j: \operatorname{Cof} \mathcal{Y} \rightarrow \mathcal{X}_{\mathcal{Y}}$. Here $\operatorname{Cof} \mathcal{Y}$ is the category of cofibrations in $\mathcal{Y}$.

Definition 1.9. We say that the inclusion functor $\iota: \mathcal{Y} \hookrightarrow \mathcal{X}$ satisfies the resolution conditions if it satisfies the following three conditions:

(Res 1) $\mathcal{Y}$ is closed under extensions in $\mathcal{X}$.

(Res 2) For any object $x$ in $\mathcal{X}$, there are an object $y$ in $\mathcal{Y}$ and a cofibration $x \longmapsto y$.

(Res 3) For any cofibration sequence $x \longmapsto y \rightarrow z$ in $\mathcal{X}$, if $y$ is in $\mathcal{Y}$, then $z$ is also in $\mathcal{Y}$.

Lemma 1.10. (cf. [13, Proof of 4.1.], [26, p.524]) If the inclusion functor $\iota: \mathcal{Y} \hookrightarrow \mathcal{X}$ satisfies the resolution conditions, then $\mathcal{X}_{\mathcal{Y}}$ is contractible.

Proof. Since Cof $\mathcal{Y}$ has the initial object, it is contractible. We intend to apply Quillen's Theorem A to $j$ : $\operatorname{Cof} \mathcal{Y} \rightarrow \mathcal{X} \mathcal{Y}$ and then we will get the result. Fix an object $a$ in $\mathcal{X}_{\mathcal{Y}}$ and objects $x$ and $y$ in $\mathcal{Y}$ such that there is a cofibration sequence $a \longmapsto x \rightarrow y$. Now we will prove that $a / j$ is contractible. To do so, consider an object $a \longmapsto b$ in $a / j$. Since $\mathcal{Y}$ is closed under extensions in $\mathcal{X}$, in the push out diagram

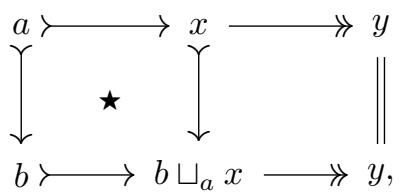

where the square $\star$ is coCartesian, we can take $b \sqcup_{a} x$ in $\mathcal{Y}$. Now there are the natural transformations

$$
((a \longmapsto b) \mapsto(a \longmapsto b)) \longmapsto\left((a \longmapsto b) \mapsto\left(a \longmapsto b \sqcup_{a} x\right)\right) \longleftrightarrow((a \longmapsto b) \mapsto(a \longmapsto x))
$$

between the identity functor and the constant functor $(a \longmapsto b) \mapsto(a \longmapsto x)$ on $a / j$. Therefore $a / j$ is contractible.

Lemma 1.11. If $\iota: \mathcal{Y} \hookrightarrow \mathcal{X}$ satisfies the resolution conditions, then for each nonnegative integer $n, \mathcal{S}_{n} \mathcal{Y} \hookrightarrow \mathcal{S}_{n} \mathcal{X}$ also satisfies the resolution conditions.

Proof. Since the filtered object categories $F_{n-1} \mathcal{X}$ and $F_{n-1} \mathcal{Y}$ are equivalent to $\mathcal{S}_{n} \mathcal{X}$ and $\mathcal{S}_{n} \mathcal{Y}$, respectively, as categories with cofibrations, we just check that the inclusion functor $F_{n} \mathcal{Y} \hookrightarrow F_{n} \mathcal{X}$ satisfies the resolution conditions. The condition (Res 1) has been proven in 1.7. We first check the condition (Res 2). For a filtered object $x_{0} \longmapsto \ldots \longmapsto x_{n}$, we have an object $y_{0}$ in $\mathcal{Y}$ and a cofibration $x_{0} \longmapsto y_{0}$ by the assumption (Res 2). For each $k<n$, if we have a filtered object $y_{0} \longmapsto \ldots \longmapsto y_{k}$ in $F_{k} \mathcal{Y}$ and a cofibration $x_{0} \longmapsto \ldots \longmapsto x_{k}$ to $y$ in $F_{k} \mathcal{Y}$, then we have an object $y_{k+1}$ in $\mathcal{Y}$ and a cofibration $y_{k} \sqcup_{x_{k}} x_{k+1} \longmapsto y_{k+1}$ by the assumption (Res 2) again. Therefore inductively, we can find a filtered object $y$ in $F_{n} \mathcal{Y}$ and a cofibration $x \longmapsto y$. Next we check the condition (Res 3). For a cofibration sequence $x \longmapsto y \rightarrow z$ in $F_{n} \mathcal{X}$, if $y$ is in $F_{n} \mathcal{Y}$, 
then by applying the assumption (Res 3 ) term-wisely, we notice that $z$ is also in $F_{n} \mathcal{Y}$.

Definition 1.12. We say that the inclusion functor $\iota: \mathcal{Y} \hookrightarrow \mathcal{X}$ satisfies the strong resolution conditions if for any non-negative integer $m, \mathcal{Y}(m, w) \hookrightarrow \mathcal{X}(m, w)$ satisfies the resolution conditions.

Theorem 1.13 (Resolution theorem). In the notation above, if $\iota$ satisfies the strong resolution conditions and $\mathcal{X}$ is essentially small, then the canonical map induced by $\iota, K(\mathcal{Y} ; w) \rightarrow K(\mathcal{X} ; w)$ is a homotopy equivalence.

Proof. We may assume that $\mathcal{X}$ is small. By [29, p.344, p.345 1.5.7], we have the sequence of homotopy type of a fibration

$$
w \mathcal{S}_{\bullet} \mathcal{Y} \stackrel{w \mathcal{S}_{\bullet} \iota}{\rightarrow} w \mathcal{S}_{\bullet} \mathcal{X} \rightarrow w \mathcal{S}_{\bullet} F_{\bullet}(\mathcal{X}, \mathcal{Y}) .
$$

Fix non-negative integers $n$ and $m$. We have the following equalities:

$$
\begin{aligned}
N_{m} w \mathcal{S}_{n} F_{\bullet}(\mathcal{X}, \mathcal{Y}) & \stackrel{\sim}{\rightarrow} f_{\bullet}\left(\mathcal{S}_{n} \mathcal{X}\left(m, w \mathcal{S}_{n} \mathcal{X}\right), \mathcal{S}_{n} \mathcal{Y}\left(m, w \mathcal{S}_{n} \mathcal{Y}\right)\right) \\
& \sim \\
\rightarrow & f_{\bullet}\left(\mathcal{S}_{n}(\mathcal{X}(m, w)), \mathcal{S}_{n}(\mathcal{Y}(m, w))\right),
\end{aligned}
$$

where $f_{\bullet}$ denote the simplicial set of objects of $F_{\bullet}$ and for the definition $\mathcal{X}(m, w)$ and so on see 1.3. By the realization lemma [23, Appendix A] or [28, 5.1], and by replacing $\mathcal{X}(m, w)$ and $\mathcal{Y}(m, w)$ with $\mathcal{X}$ and $\mathcal{Y}$, respectively, we shall just check the following claim:

Claim. For a small category with cofibrations $\mathcal{X}$ and $\iota: \mathcal{Y} \hookrightarrow \mathcal{X}$ a full sub category closed under extensions. Assume that $\iota$ is satisfying the resolution conditions. Then for each non-negative integer $n, f_{\bullet}\left(\mathcal{S}_{n} \mathcal{X}, \mathcal{S}_{n} \mathcal{Y}\right)$ is contractible.

If $n=0$, this claim is trivial. For $n \geqslant 1$, by 1.11 and by replacing $\mathcal{S}_{n} \mathcal{X}$ and $\mathcal{S}_{n} \mathcal{Y}$ with $\mathcal{X}$ and $\mathcal{Y}$, respectively, we shall assume $n=1$. Now $f_{\bullet}(\mathcal{X}, \mathcal{Y})$ is just the nerve of $\mathcal{X}_{\mathcal{Y}}$ in 1.8 and therefore we get the result by 1.10 .

\section{Semi-direct products of exact categories}

In this section, we will establish the theory of semi-direct products of exact categories (with weak equivalences) which is a generalization of $[\mathbf{2 0}, \S 3]$. Let us start by preparing the general terminologies about cubes. Let $S$ be a set, $\mathcal{C}$ a category, $\mathcal{A}$ an abelian category and $R$ a commutative ring with 1.

Definition 2.1. We define the category of $S$-cubes in $\mathcal{C}$ by

$$
\operatorname{Cub}^{S}(\mathcal{C}):=\mathcal{H O} \mathcal{M}\left(\mathcal{P}(S)^{o p}, \mathcal{C}\right) .
$$

An object in $\mathbf{C u b}^{S}(\mathcal{C})$ is said to be an $S$-cube. Let $x$ be an $S$-cube in $\mathcal{C}$. For $T \in \mathcal{P}(S)$ and $k \in T$, we denote $x(T)$ by $x_{T}$ and call it a vertex of $x$ (at $T$ ) and we also write $d_{T}^{x, k}$ or shortly $d_{T}^{k}$ for $x(T \backslash\{k\} \hookrightarrow T)$ and call it a (k-direction) boundary morphism of $x$. 
Remark 2.2. For a positive integer $n$, we have the canonical category isomorphism

$$
\mathcal{P}((n]) \stackrel{\sim}{\rightarrow}[1]^{\times n}, \quad S \mapsto\left(\chi_{S}(k)\right),
$$

where $\chi_{S}$ is the characteristic function associated with $S$. Namely $\chi_{S}(k)=1$ if $k$ is in $S$ and otherwise $\chi_{S}(k)=0$. Through the isomorphism above, we consider $(n]$-cubes to be contravariant functors from $[1]^{\times n}$ and call them $n$-cubes. $\mathbf{C u b}^{(n]}$ is abbreviated to $\mathbf{C u b}^{n}$.

Remark 2.3. For any abelian (resp. exact) category $\mathcal{C}$ and a set $S, \mathbf{C} \mathbf{u b}^{S}(\mathcal{C})$ is an abelian category (resp. exact category by defining the admissible exact sequences to be termwise admissible exact sequences in $\mathcal{C}$ ).

Remark 2.4. For a pair of disjoint sets $S$ and $T$, we have the category isomorphism

$$
\mathcal{P}(S) \times \mathcal{P}(T) \stackrel{\sim}{\rightarrow} \mathcal{P}(S \sqcup T),(U, V) \mapsto U \cup V
$$

and by the exponential law, the isomorphism above induces the category isomorphism

$$
\mathbf{C u b}^{S \sqcup T}(\mathcal{C}) \stackrel{\sim}{\rightarrow} \mathbf{C u b}^{S}\left(\mathbf{C u b}^{T}(\mathcal{C})\right) .
$$

Moreover if $\mathcal{C}$ is an abelian (resp. exact) category, then the isomorphism above is an exact functor.

Definition 2.5 (Homology of cubes). Let us fix an $S$-cube $x$ in $\mathcal{A}$. For each $k$, the $k$-direction 0 -th (resp. 1-th) homology of $x$ is an $S \backslash\{k\}$-cube in $\mathcal{A}$ denoted by $\mathrm{H}_{0}^{k}(x)$ (resp. $\left.\mathrm{H}_{1}^{k}(x)\right)$ and defined by $\mathrm{H}_{0}^{k}(x)_{T}:=$ Coker $d_{T \cup\{k\}}^{k}\left(\right.$ resp. $\mathrm{H}_{1}^{k}(x)_{T}:=\operatorname{Ker} d_{T \cup\{k\}}^{k}$ ).

The following lemma is sometimes useful to deal with morphisms of cubes:

Lemma 2.6. We have the following assertions:

(1) For any $S$-cube $x$, every $T, U \in \mathcal{P}(S)$ such that $T \subset U$ and $U \backslash T$ is a finite set, the morphism $x(T \subset U)$ is described as compositions of boundary morphisms.

(2) Assume that $S$ is a finite set. For any $S$-cubes $x$, $y$ and a family of morphisms $f=\left\{f_{T}: x_{T} \rightarrow y_{T}\right\}_{T \in \mathcal{P}(S)}$ in $\mathcal{C}, f: x \rightarrow y$ is a morphism of $S$-cubes in $\mathcal{C}$ if and only if for any $T \in \mathcal{P}(S)$ and $k \in T$, we have the equality $d_{T}^{y, k} f_{T}=f_{T \backslash\{k\}} d_{T}^{x, k}$.

Example 2.7 (Typical cubes). Assume that $S$ is a finite set and let $\mathfrak{f}_{S}=\left\{f_{s}\right\}_{s \in S}$ be a family of elements in $R$. The typical cube associated with $\left\{f_{s}\right\}_{s \in S}$ is an $S$-cube in $\mathcal{P}_{R}$ denoted by $\operatorname{Typ}_{R}\left(\mathfrak{f}_{S}\right)$ and defined by $\operatorname{Typ}_{R}\left(\mathfrak{f}_{S}\right)_{T}=R$ and $d_{T}^{\operatorname{Typ}_{R}\left(\mathfrak{f}_{S}\right), t}=f_{t}$ for any $T \in \mathcal{P}(S)$ and $t \in T$.

The following lemma is often used when we are dealing with cubes. Its proof is very easy: 
Lemma 2.8 (Cube lemma). For the diagram below in a category $\mathcal{C}$

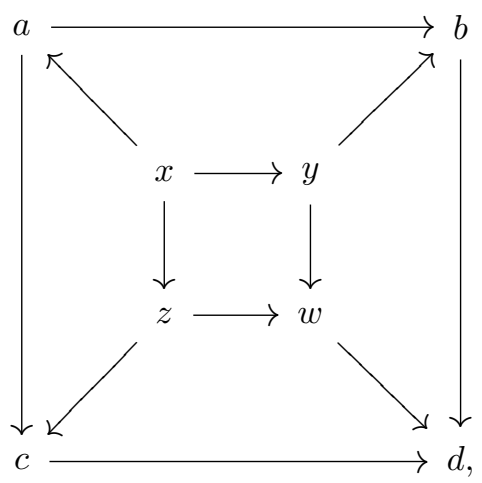

assume that the morphism $\overrightarrow{w d}$ is a monomorphism (resp. $\overrightarrow{x a}$ is an epimorphism) and every square except xywz (resp. abdc) is commutative. Then xywz (resp. abdc) is also commutative.

Definition 2.9. Let $\mathcal{E}$ and $\mathcal{F}$ be full strict exact subcategories of $\mathcal{A}$. We define the category $\mathcal{F} \ltimes \mathcal{E}$ as follows. $\mathcal{F} \ltimes \mathcal{E}$ is the full subcategory of $\mathbf{C u b}^{1}(\mathcal{E})$ of those morphisms $x_{1} \rightarrow x_{0}$ in $\mathcal{E}$ which are monomorphisms with $\mathcal{A}$-cokernel in $\mathcal{F}$.

Proposition 2.10. In the notations above, if $\mathcal{F}$ satisfies either condition (1) or (2) below, then $\mathcal{F} \ltimes \mathcal{E}$ is a full strict exact subcategory of $\operatorname{Cub}^{1}(\mathcal{A})$. Moreover, $\mathrm{H}_{0}: \mathcal{F} \ltimes \mathcal{E} \rightarrow \mathcal{F}$ is exact.

(1) $\mathcal{F}$ is closed under extensions, that is, for an exact sequence $a \longmapsto b \rightarrow c$ in $\mathcal{A}$, if $a$ and $c$ are isomorphic to objects in $\mathcal{F}$, respectively, then $b$ is also isomorphic to an object in $\mathcal{F}$.

(2) $\mathcal{F}$ is closed under admissible sub- and quotient objects, that is, for an exact sequence $a \longmapsto b \rightarrow c$ in $\mathcal{A}$, if $b$ is isomorphic to an object in $\mathcal{F}$, then $a$ and $c$ are also isomorphic to objects in $\mathcal{F}$, respectively.

Proof. We may assume that $\mathcal{E}$ and $\mathcal{F}$ are closed under isomorphisms in $\mathcal{A}$. That is, if $a$ is an object in $\mathcal{A}$ which is isomorphic to an object in $\mathcal{E}$ (resp. $\mathcal{F}$ ), then $a$ is also in $\mathcal{E}$ (resp. $\mathcal{F}$ ). We declare that a sequence $x \rightarrow y \rightarrow z$ in $\mathcal{F} \ltimes \mathcal{E}$ is an admissible exact sequence if it is exact in $\mathbf{C u b}^{1}(\mathcal{A})$. Obviously a split short exact sequence in $\mathcal{F} \ltimes \mathcal{E}$ is an admissible exact sequence. We need to prove that the class of admissible monomorphisms (resp. admissible epimorphisms) is closed under compositions and co-base change (resp. base change) along arbitrary morphisms. We just check for the admissible monomorphisms case. To prove for the admissible epimorphisms case is similar. For a pair of composable admissible monomorphisms $x \longmapsto y \longmapsto z$, by the snake lemma in $\mathbf{C u b}^{1}(\mathcal{A})$, we have the short exact sequence

$$
y / x \longmapsto z / x \rightarrow z / y
$$


in $\mathbf{C u b}^{1}(\mathcal{A})$. Applying the snake lemma to the following diagram

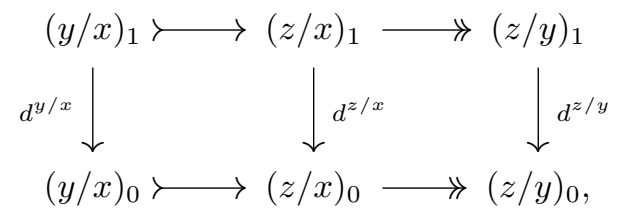

we learn that $d^{z / x}$ is a monomorphism in $\mathcal{A}$. Now let us assume the condition (1) (resp. (2)). Then by considering the following short exact sequence in $\mathcal{A}$

$$
\begin{gathered}
\mathrm{H}_{0}(y / x) \longmapsto \mathrm{H}_{0}(z / x) \rightarrow \mathrm{H}_{0}(z / y), \\
\left(\text { resp. } \mathrm{H}_{0}(x) \longmapsto \mathrm{H}_{0}(z) \rightarrow \mathrm{H}_{0}(z / x),\right)
\end{gathered}
$$

we notice that $\mathrm{H}_{0}(z / x)$ is actually in $\mathcal{F}$. Therefore the short exact sequence

$$
x \longmapsto z \rightarrow z / x
$$

is an admissible exact sequence in $\mathcal{F} \ltimes \mathcal{E}$. Hence the class of admissible monomorphisms is closed under compositions. Next let us consider morphisms $y \leftarrow x \longmapsto z$ in $\mathcal{F} \ltimes \mathcal{E}$. Consider the coproduct $y \sqcup_{x} z$ in $\operatorname{Cub}^{1}(\mathcal{A})$. We have the following pushout diagram

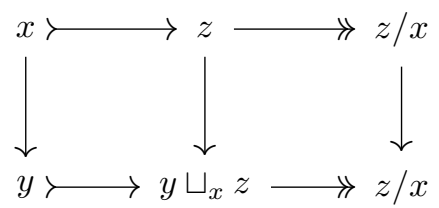

in $\mathbf{C u b}^{1}(\mathcal{A})$. Then since the class of admissible monomorphisms in $\mathcal{E}$ is closed under the co-base change by arbitrary morphisms, $\left(y \sqcup_{x} z\right)_{i}$ is in $\mathcal{E}$ for $i=0,1$. Applying the snake lemma to the following diagram

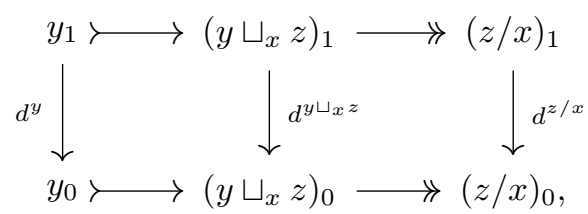

we learn that $d^{y \sqcup_{x} z}$ is a monomorphism in $\mathcal{A}$ and by applying the functor $\mathrm{H}_{0}$ to the pushout diagram (1) above, we get the following commutative diagram

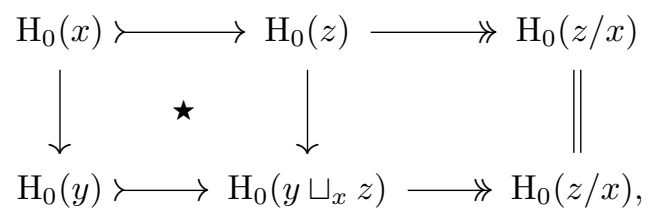

where the horizontal lines are short exact sequences in $\mathcal{A}$. Thus $\star$ is coCartesian and since the class of admissible monomorphisms in $\mathcal{F}$ is closed under co-base change by 
arbitrary morphisms, $\mathrm{H}_{0}\left(y \sqcup_{x} z\right)$ is in $\mathcal{F}$. We conclude that $y \sqcup_{x} z$ is in $\mathcal{F} \ltimes \mathcal{E}$ and

$$
y \longmapsto y \sqcup_{x} z \rightarrow z / x
$$

is an admissible exact sequences in $\mathcal{F} \ltimes \mathcal{E}$.

Definition 2.11. In the situation 2.10 , moreover assume that $\mathcal{F}$ has the subcategory $w=w(\mathcal{F})$ containing the class of isomorphisms in $\mathcal{F}$. We define the subcategory $t w(\mathcal{F} \ltimes \mathcal{E})$ of $\mathcal{F} \ltimes \mathcal{E}$ as follows. A morphism $f: x \rightarrow y$ in $\mathcal{F} \ltimes \mathcal{E}$ is in $t w(\mathcal{F} \ltimes \mathcal{E})$ if and only if $\mathrm{H}_{0}(f): \mathrm{H}_{0}(x) \rightarrow \mathrm{H}_{0}(y)$ is in $w(\mathcal{F})$. Then every isomorphism in $\mathcal{F} \ltimes \mathcal{E}$ is in $t w(\mathcal{F} \ltimes \mathcal{E})$.

Proposition 2.12. In the notation above, if $w(\mathcal{F})$ satisfies the gluing (resp. cogluing, saturational, extensional) axiom, then $t w(\mathcal{F} \ltimes \mathcal{E})$ also does. In particular if $(\mathcal{F}, w)$ is a Waldhausen exact category, then $(\mathcal{F} \ltimes \mathcal{E}, t w(\mathcal{F} \ltimes \mathcal{E}))$ is also a Waldhausen exact category and the functor $\mathrm{H}_{0}:(\mathcal{F} \ltimes \mathcal{E}, t w(\mathcal{F} \ltimes \mathcal{E})) \rightarrow(\mathcal{F}, w)$ is a morphism of Waldhausen categories.

Proposition 2.13. In the notation 2.12, moreover assume that $(\mathcal{F}, w)$ is a Waldhausen category and $\mathcal{F}$ is contained in $\mathcal{E}$, then the functor $\mathrm{H}_{0}$ induces a homotopy equivalence on $K$-theory:

$$
K\left(\mathrm{H}_{0}\right): K(\mathcal{F} \ltimes \mathcal{E} ; t w) \rightarrow K(\mathcal{F} ; w) .
$$

Proof. Define the morphism of Waldhausen categories $s:(\mathcal{F}, w) \rightarrow(\mathcal{F} \ltimes \mathcal{E}, t w)$ by $x \mapsto[0 \rightarrow x]$. Then obviously we have the equality $\mathrm{H}_{0} \circ s=$ id. Moreover there is the natural weak equivalence id $\rightarrow s \circ \mathrm{H}_{0}$ defined by the canonical morphisms

$$
\left[\begin{array}{c}
x_{1} \\
d^{x} \downarrow \\
x_{0}
\end{array}\right] \rightarrow\left[\begin{array}{c}
0 \\
\downarrow \\
\downarrow \\
\mathrm{H}_{0}(x)
\end{array}\right]
$$

for any object $x$ in $\mathcal{F} \ltimes \mathcal{E}$. Therefore by [29, p.330 1.3.1], we learn that $w S_{\bullet} \mathrm{H}_{0}$ is a homotopy equivalence.

Next let $\mathcal{E}_{i}$ and $\mathcal{F}_{i}(i=1,2)$ be full strict exact subcategories of $\mathcal{A}$. Moreover we suppose that $\mathcal{F}_{i}$ satisfies either condition (1) or (2) in 2.10. Then by $2.10, \mathcal{F}_{i} \ltimes \mathcal{E}_{i}$ is an exact category.

Proposition 2.14. Assume that the inclusion functors $\mathcal{E}_{1} \hookrightarrow \mathcal{E}_{2}$ and $\mathcal{F}_{1} \hookrightarrow \mathcal{F}_{2}$ are closed under extensions. That is, for an admissible exact sequence

$$
x \longmapsto y \rightarrow z
$$

in $\mathcal{E}_{2}$ (resp. $\left.\mathcal{F}_{2}\right)$ if $x$ and $z$ are isomorphic to objects in $\mathcal{E}_{1}$ (resp. $\mathcal{F}_{1}$ ), then $y$ is also in $\mathcal{E}_{1}$ (resp. $\left.\mathcal{F}_{1}\right)$. Then $\mathcal{F}_{1} \ltimes \mathcal{E}_{1} \hookrightarrow \mathcal{F}_{2} \ltimes \mathcal{E}_{2}$ is also closed under extensions.

Proposition 2.15. Assume that the inclusion functors $\mathcal{E}_{1} \hookrightarrow \mathcal{E}_{2}$ and $\mathcal{F}_{1} \hookrightarrow \mathcal{F}_{2}$ are closed under taking kernels of admissible epimorphisms. That is, for a short exact 
sequence

$$
x \longmapsto y \rightarrow z
$$

in $\mathcal{A}$ if $z$ is isomorphic to an object in $\mathcal{E}_{2}$ (resp. $\mathcal{F}_{2}$ ) and $y$ is isomorphic to an object in $\mathcal{E}_{1}\left(\right.$ resp. $\left.\mathcal{F}_{1}\right)$, then $x$ is also isomorphic to an object in $\mathcal{E}_{1}$ (resp. $\left.\mathcal{F}_{1}\right)$. Then $\mathcal{F}_{1} \ltimes \mathcal{E}_{1} \hookrightarrow \mathcal{F}_{2} \ltimes \mathcal{E}_{2}$ is also closed under taking kernels of admissible epimorphisms.

Proof of 2.14 and 2.15. Let us consider the short exact sequence below in $\mathbf{C u b}^{1}(\mathcal{A})$

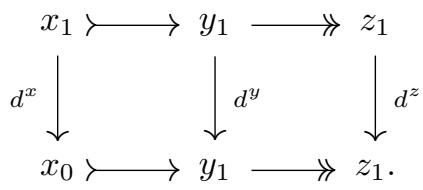

If $d^{z}$ and $d^{x}$ are monomorphisms (resp. $d^{y}$ is a monomorphism), then $d^{y}$ (resp. $d^{x}$ ) is also. In this case, observing the $3 \times 3$ commutative diagram below

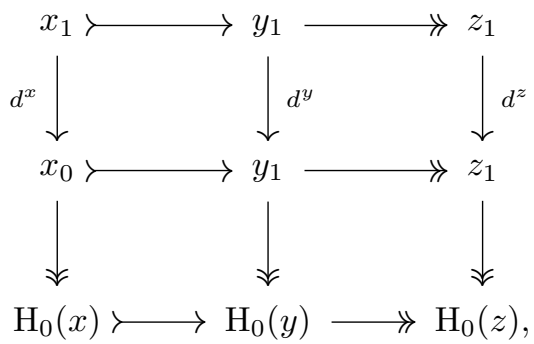

we learn that if the condition 2.14 (resp. 2.15) is verified and if $z$ is isomorphic to an object in $\mathcal{F}_{1} \ltimes \mathcal{E}_{1}$ (resp. $\mathcal{F}_{2} \ltimes \mathcal{E}_{2}$ ) and if $x$ (resp. $y$ ) is isomorphic to an object in $\mathcal{F}_{1} \ltimes \mathcal{E}_{1}$, then $y$ (resp. $x$ ) is also isomorphic to an object in $\mathcal{F}_{1} \ltimes \mathcal{E}_{1}$.

Remark 2.16. The assertions 2.14, 2.15 and its dual imply the following statements:

(1) Assume that the inclusion functors $\mathcal{E}_{1} \hookrightarrow \mathcal{E}_{2}$ and $\mathcal{F}_{1} \hookrightarrow \mathcal{F}_{2}$ are closed under admissible sub- and quotient objects, then $\mathcal{F}_{1} \ltimes \mathcal{E}_{1}$ is also closed in $\mathcal{F}_{2} \ltimes \mathcal{E}_{2}$.

(2) Let $\mathcal{E}, \mathcal{F}$ be full subcategories of $\mathcal{A}$ closed under extensions in $\mathcal{A}$. Then $\mathcal{F} \ltimes \mathcal{E}$ is closed under extensions in $\mathbf{C u b}^{1}(\mathcal{A})$.

Proposition 2.17. In the notation above, moreover let us assume the following two conditions:

(1) $\mathcal{F}_{2}$ is a full subcategory of $\mathcal{E}_{2}$.

(2) Every object in $\mathcal{E}_{1}$ is a projective object in $\mathcal{E}_{2}$ and every object in $\mathcal{F}_{1}$ is a projective object in $\mathcal{F}_{2}$.

Then all objects in $\mathcal{F}_{1} \ltimes \mathcal{E}_{1}$ are projective objects in $\mathcal{F}_{2} \ltimes \mathcal{E}_{2}$. 
Proof. Let us consider the left diagram in $\mathcal{F}_{2} \ltimes \mathcal{E}_{2}$ below
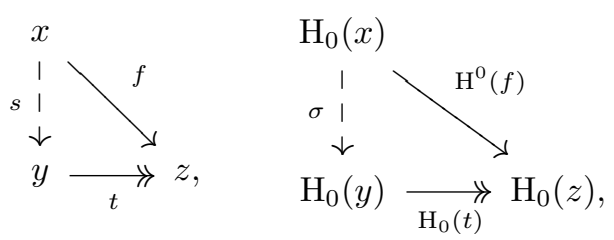

where $x$ is an object in $\mathcal{F}_{1} \ltimes \mathcal{E}_{1}$ and $t$ is an admissible epimorphism. Then by applying $\mathrm{H}_{0}$ to the diagram, we get the right diagram in $\mathcal{F}_{2}$ above. Since $\mathrm{H}_{0}(x)$ is a projective object in $\mathcal{F}_{2}$, there is a morphism $\sigma: \mathrm{H}_{0}(x) \rightarrow \mathrm{H}_{0}(y)$ which makes the right diagram above commutative.

Claim. There is a morphism $s^{\prime}: x \rightarrow y$ such that $\mathrm{H}_{0}\left(s^{\prime}\right)=\sigma$ and $t s^{\prime}$ is chain homotopic to $f$.

Proof of claim. Let us consider the left diagram of admissible exact sequences below
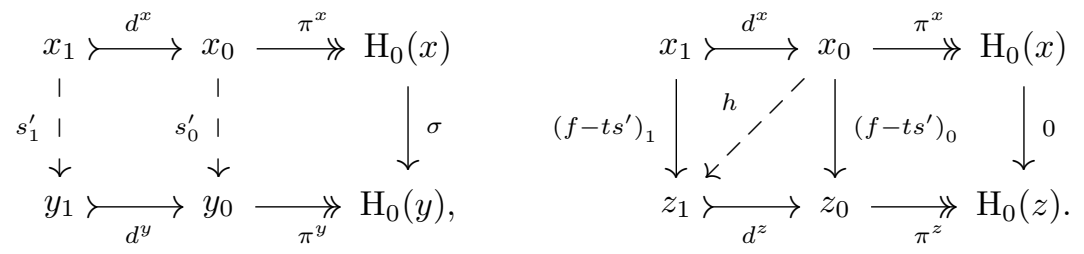

Since $x_{0}$ is a projective object in $\mathcal{E}_{2}$, we have a morphism $s_{0}^{\prime}: x_{0} \rightarrow y_{0}$ which makes the diagram above commutative. Therefore by the universality for the kernel of $d^{y}$, we also have a morphism $s_{1}^{\prime}: x_{1} \rightarrow y_{1}$ in the left commutative diagram above. Then we have the equalities $\mathrm{H}_{0}(f)=\mathrm{H}_{0}(t) \sigma=\mathrm{H}_{0}\left(t s^{\prime}\right)$. Therefore we have $\pi^{z}\left(f-t s^{\prime}\right)_{0}=0$. By the universality for the kernel of $\pi^{z}$, we have a morphism $h: x_{0} \rightarrow z_{1}$ such that $\left(f-t s^{\prime}\right)_{0}=d^{z} h$. Since $d^{z}$ is a monomorphism, we also have the equality $\left(f-t s^{\prime}\right)_{1}=$ $h d^{x}$. Hence we get the desired result.

Since $x_{0}$ is a projective object in $\mathcal{E}_{2}$, we have a morphism $u: x_{0} \rightarrow y_{1}$ such that $t_{1} u=h$.

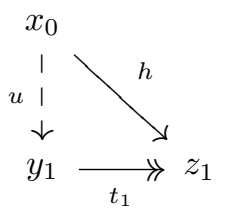

We put $s_{1}:=s_{1}^{\prime}+u d^{x}$ and $s_{0}:=s_{0}^{\prime}+d^{y} u$. Then we can easily check that $s$ is a morphism of complexes and $f=t s$.

Definition 2.18. Let $\mathcal{H} \hookrightarrow \mathcal{G}$ be strict exact subcategories of $\mathcal{A}$. Assume that $\mathcal{G}$ satisfies either condition (1) or (2) in 2.10. Moreover assume that $\mathcal{G}$ has a class of weak equivalences $w \mathcal{G}$ which satisfies the axioms of weak equivalences in [29]. We put $v:=w \mathcal{G} \cap \mathcal{H}$ and it is a class of weak equivalences in $\mathcal{H}$. We define the new class of weak equivalences $l v(\mathcal{G} \ltimes \mathcal{H})$ in $\mathcal{G} \ltimes \mathcal{H}$ as follows. A morphism $f: x \rightarrow y$ in $\mathcal{G} \ltimes \mathcal{H}$ is 
in $l v(\mathcal{G} \ltimes \mathcal{H})$ if and only if $f_{i}: x_{i} \rightarrow y_{i}$ for $i=0,1$ is in $v \mathcal{H}$. We call a morphism in $l v(\mathcal{G} \ltimes \mathcal{H})$ a level weak equivalence.

We can easily check that $(\mathcal{G} \ltimes \mathcal{H}, l v)$ is a Waldhausen category.

Proposition 2.19 (Abstract split fibration theorem). Let $\mathcal{G}, \mathcal{H}, w$ and $v$ be as in 2.18. Then we have split fibration sequences

$$
\begin{gathered}
K(\mathcal{H} ; v) \rightarrow K(\mathcal{G} \ltimes \mathcal{H} ; l v) \rightarrow K(\mathcal{G} ; w) \text { and } \\
K(\mathcal{H}) \rightarrow K(\mathcal{G} \ltimes \mathcal{H}) \rightarrow K(\mathcal{G}) .
\end{gathered}
$$

Proof. Let us denote the category of acyclic complexes in $\mathcal{G} \ltimes \mathcal{H}$ by $(\mathcal{G} \ltimes \mathcal{H})^{q}$. Since $(\mathcal{G} \ltimes \mathcal{H})^{q}$ is closed under extensions in $\mathcal{G} \ltimes \mathcal{H}$, it naturally becomes a Waldhausen category and the association $x \mapsto\left[x \stackrel{\mathrm{id}_{x}}{\rightarrow} x\right]$ gives an equivalence between $\mathcal{H}$ and $(\mathcal{G} \ltimes \mathcal{H})^{q}$ as Waldhausen categories. On the other hand, there is an equivalence of Waldhausen categories

$$
\mathcal{G} \ltimes \mathcal{H} \stackrel{\sim}{\rightarrow} \mathrm{E}\left((\mathcal{G} \ltimes \mathcal{H})^{q}, \mathcal{G} \ltimes \mathcal{H}, \mathcal{G}\right)
$$

$$
x \mapsto\left(\left[\begin{array}{c}
x_{1} \\
\operatorname{id}_{x_{1}} \downarrow \\
x_{1}
\end{array}\right] \underset{d^{x}}{\stackrel{\mathrm{id}_{x_{1}}}{\rightarrow}}\left[\begin{array}{c}
x_{1} \\
d^{x} \downarrow \\
x_{0}
\end{array}\right] \rightarrow \begin{array}{c}
\rightarrow \\
\\
\end{array}\right]
$$

where $\mathrm{E}\left((\mathcal{G} \ltimes \mathcal{H})^{q}, \mathcal{G} \ltimes \mathcal{H}, \mathcal{G}\right)$ is the exact category of admissible exact sequences $x \longmapsto y \rightarrow z$ in $\mathcal{G} \ltimes \mathcal{H}$ such that $x$ is in $(\mathcal{G} \ltimes \mathcal{H})^{q}$ and $z$ is in $\mathcal{G}$. Moreover $\mathrm{E}\left((\mathcal{G} \ltimes \mathcal{H})^{q}\right.$, $\mathcal{G} \ltimes \mathcal{H}, \mathcal{G}$ ) has the natural class of weak equivalences $l v$ induced from $\mathcal{G} \ltimes \mathcal{H}$. Hence by the additivity theorem in [29, Proposition 1.3.2.], we get the first fibration sequence. The second fibration sequence is given by taking $w=i_{\mathcal{G}}$ and $v=i_{\mathcal{H}}$ the class of all isomorphisms in $\mathcal{G}$ and $\mathcal{H}$ and by the equality $l i_{\mathcal{H}}=i_{\mathcal{G} \ltimes \mathcal{H}}$ in the first situation.

Definition 2.20 (Adroit systems). An adroit (resp. a strongly adroit) system in an abelian category $\mathcal{A}$ is a triple $\mathcal{X}=\left(\mathcal{E}_{1}, \mathcal{E}_{2}, \mathcal{F}\right)$ consisting of strict exact subcategories $\mathcal{E}_{1} \hookrightarrow \mathcal{E}_{2} \hookleftarrow \mathcal{F}$ of $\mathcal{A}$ such that they satisfies the following conditions (Adr 1), (Adr 2), (Adr 3) and (Adr 4) (resp. (Adr 1), (Adr 2), (Adr 3) and (Adr 5)):

(Adr 1) $\mathcal{F} \ltimes \mathcal{E}_{1}$ and $\mathcal{F} \ltimes \mathcal{E}_{2}$ are strict exact subcategories of $\mathbf{C u b}^{1} \mathcal{A}$.

(Adr 2) $\mathcal{E}_{1}$ is closed under extensions in $\mathcal{E}_{2}$.

(Adr 3) Let $x \longmapsto y \rightarrow z$ be an admissible exact sequence in $\mathcal{A}$. Assume that $y$ is isomorphic to an object in $\mathcal{E}_{1}$ and $z$ is isomorphic to an object in either $\mathcal{E}_{1}$ or $\mathcal{F}$. Then $x$ is isomorphic to an object in $\mathcal{E}_{1}$.

(Adr 4) For any object $z$ in $\mathcal{E}_{2}$, there is an object $y$ in $\mathcal{E}_{1}$ and an admissible epimorphism $y \rightarrow z$ in $\mathcal{E}_{2}$.

(Adr 5) For any non-negative integer $m$ and any object $z$ in $\mathcal{H O M}\left([m], \mathcal{E}_{2}\right)$, there is an object $y$ in $\mathcal{H O M}\left([m], \mathcal{E}_{1}\right)$ and an admissible epimorphism $y \rightarrow z$ in $\mathcal{H O M}\left([m], \mathcal{E}_{2}\right)$. 
Theorem 2.21. Let $\mathcal{X}=\left(\mathcal{E}_{1}, \mathcal{E}_{2}, \mathcal{F}\right)$ be a triple of strict exact subcategories $\mathcal{E}_{1} \hookrightarrow \mathcal{E}_{2} \hookleftarrow \mathcal{F}$ of $\mathcal{A}$ and $w$ a class of morphisms in $\mathcal{F}$ such that $(\mathcal{F}, w)$ is a Waldhausen exact category. Then

(1) If the triple $\mathcal{X}$ is an adroit (resp. a strongly adroit) system, then the inclusion functor of opposite categories

$$
\left(\mathcal{F} \ltimes \mathcal{E}_{1}\right)^{o p} \hookrightarrow\left(\mathcal{F} \ltimes \mathcal{E}_{2}\right)^{o p}\left(\text { resp. }\left(\left(\mathcal{F} \ltimes \mathcal{E}_{1}\right)^{o p}, t w^{o p}\right) \hookrightarrow\left(\left(\mathcal{F} \ltimes \mathcal{E}_{2}\right)^{o p}, t w^{o p}\right)\right)
$$

satisfies the resolution (resp. strong resolution) conditions in 1.12. In particular we have a homotopy equivalence on $K$-theory:

$$
\begin{gathered}
K\left(\mathcal{F} \ltimes \mathcal{E}_{1}\right) \rightarrow K\left(\mathcal{F} \ltimes \mathcal{E}_{2}\right) \\
\left(\text { resp. } K\left(\mathcal{F} \ltimes \mathcal{E}_{1} ; t w\right) \rightarrow K\left(\mathcal{F} \ltimes \mathcal{E}_{2} ; t w\right)\right) .
\end{gathered}
$$

(2) (Abstract weight declension theorem). If the triple $\mathcal{X}$ is a strongly adroit system, then the exact functor $\mathrm{H}_{0}:\left(\mathcal{F} \ltimes \mathcal{E}_{1}, t w\right) \rightarrow(\mathcal{F}, w)$ induces a homotopy equivalence on $K$-theory:

$$
K\left(\mathrm{H}_{0}\right): K\left(\mathcal{F} \ltimes \mathcal{E}_{1} ; t w\right) \rightarrow K(\mathcal{F} ; w) .
$$

(3) If the triple $\mathcal{X}$ is an adroit system, then for $i=1$, 2, the exact functor $\theta_{i}: \mathcal{F} \ltimes \mathcal{E}_{i} \rightarrow \mathcal{F} \times \mathcal{E}_{i}$ which sends a morphism $x=\left[x_{1} \stackrel{f}{\rightarrow} x_{0}\right]$ to an ordered pair $\left(\mathrm{H}_{0}(x), x_{1}\right)$ gives a homotopy equivalence on $K$-theory:

$$
K\left(\mathcal{F} \ltimes \mathcal{E}_{i}\right) \rightarrow K(\mathcal{F}) \times K\left(\mathcal{E}_{i}\right) .
$$

Proof. Proof of assertion (1): Let us fix a non-negative integer $m$. We will only prove that the inclusion functor $\left(\mathcal{F} \ltimes \mathcal{E}_{1}(m, t w)\right)^{o p} \hookrightarrow\left(\mathcal{F} \ltimes \mathcal{E}_{2}(m, t w)\right)^{o p}$ satisfies the resolution conditions in 1.9 when $\mathcal{X}$ is a strongly adroit system. When $m=0$, this yields the case when $\mathcal{X}$ is adroit. The condition (Res $\mathbf{1}$ ) follows from 1.7 and 2.14. We can easily check the condition (Res $\mathbf{3}$ ) from assumption (Adr 3) and 1.4. Next we check the condition (Res 2). For each $x$ in $\mathcal{F} \ltimes \mathcal{E}_{2}(m, w)$, by assumption (Adr 5), we have an object $y_{0}$ in $\mathcal{H O M}\left([m], \mathcal{E}_{1}\right)$ and an admissible epimorphism $y_{0} \rightarrow x_{0}$. Then for each $0 \leqslant i \leqslant m$, we put $y_{1}(i):=\operatorname{Ker}\left(y_{0}(i) \rightarrow x_{0}(i) \rightarrow \mathrm{H}_{0} x(i)\right)$. We have the following commutative diagram:

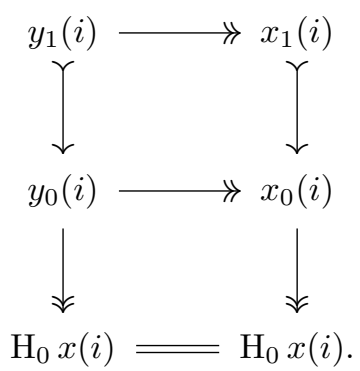

By assumption (Adr 3), we notice that $y$ is in $\mathcal{H O M}\left([m], \mathcal{F} \ltimes \mathcal{E}_{1}\right)$. Since the morphism $y(i) \rightarrow x(i)$ is a quasi-isomorphism for each $0 \leqslant i \leqslant m$, we learn that $y$ is in $\mathcal{F} \ltimes \mathcal{E}_{1}(m, w)$. Therefore we get the result. 
Proof of assertion (2): We have the factorization

$$
K\left(\mathrm{H}_{0}\right): K\left(\mathcal{F} \ltimes \mathcal{E}_{1} ; t w\right) \stackrel{\mathrm{I}}{\rightarrow} K\left(\mathcal{F} \ltimes \mathcal{E}_{2} ; t w\right) \stackrel{\mathrm{II}}{\rightarrow} K(\mathcal{F} ; w),
$$

where the maps I and II are homotopy equivalences by (1) and 2.13, respectively.

Proof of assertion (3): The inclusion functors $\mathcal{F} \ltimes \mathcal{E}_{1} \hookrightarrow \mathcal{F} \ltimes \mathcal{E}_{2}$ and $\mathcal{E}_{1} \hookrightarrow \mathcal{E}_{2}$ induce the commutative diagram below:

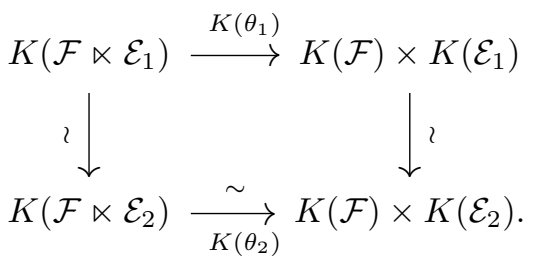

Here the vertical maps are homotopy equivalences by (1) and the resolution theorem 1.13 and the bottom map $K\left(\theta_{2}\right)$ is a homotopy equivalence by 2.19 . Therefore the map $K\left(\theta_{1}\right)$ is also a homotopy equivalence.

\section{Admissible cubes}

In this section we define and study the notion of an admissible cube in an abelian category which is a categorical variant of the concept about regular sequences. We calculate the homologies of the total complexes of admissible cubes in 3.13 and as its applications, we give a characterization of admissible cubes in terms of their faces and total complexes as in 3.15 and an inductive characterization of admissibility as in 3.16. Finally by utilizing the notion of admissibility, we extend semi-direct products to multi semi-direct products of exact categories as in 3.18. Let us start by organizing the general phraseologies of cubes. Let $\mathcal{A}$ be an abelian category.

Definition 3.1 (Restriction of cubes). Let $U, V$ be a pair of disjoint subsets of $S$. We define the functor $i_{U}^{V}: \mathcal{P}(U) \rightarrow \mathcal{P}(S), A \mapsto A \cup V$ and it induces the natural transformation $\left(i_{U}^{V}\right)^{*}: \mathbf{C u b}^{S} \rightarrow \mathbf{C u b}{ }^{U}$. For any $S$-cube $x$ in a category $\mathcal{C}$, we write $\left.x\right|_{U} ^{V}$ for $\left(i_{U}^{V}\right)^{*} x$ and it is called restriction of $x$ (to $U$ along $V$ ).

Example 3.2 (Faces of cubes). For any $S$-cube $x$ in a category $\mathcal{C}$ and $k \in S,\left.x\right|_{S \backslash\{k\}} ^{\{k\}}$, $\left.x\right|_{S \backslash\{k\}} ^{\emptyset}$ are called the backside $k$-face of $x$, the frontside $k$-face of $x$, respectively. By a face of $x$, we mean any backside or frontside $k$-face of $x$.

Recall the definition of $\mathrm{H}_{0}^{u}$ and $\mathrm{H}_{1}^{u}$ for cubes from 2.5.

Lemma 3.3. For any $S$-cube $x$ in an abelian category and any pair of disjoint subsets $U$ and $V$ and any element $u$ in $U$, we have

$$
\mathrm{H}_{p}^{u}\left(\left.x\right|_{U} ^{V}\right)=\left.\mathrm{H}_{p}^{u}(x)\right|_{U \backslash\{u\}} ^{V} \quad \text { for } p=0,1 .
$$

Proof. We will only prove the equation (2) for $p=0$. For any subset $T$ of $U \backslash\{u\}$, 
the following equalities show the equation (2) for $p=0$ :

$$
\begin{aligned}
\left.\mathrm{H}_{0}^{u}\left(\left.x\right|_{U} ^{V}\right)\right|_{T} & =\operatorname{Coker}\left(\left(\left.x\right|_{U} ^{V}\right)_{T \sqcup\{u\}} \stackrel{d_{T \sqcup\{u\}}^{x \mid V}, u}{\longrightarrow}\left(\left.x\right|_{U} ^{V}\right)_{T}\right) \\
& =\operatorname{Coker}\left(x_{V \sqcup T \sqcup\{u\}} \stackrel{d_{V \sqcup T \sqcup\{, u\}}^{x, u}}{\rightarrow} x_{V \sqcup T}\right)=\mathrm{H}_{0}^{u}(x)_{V \sqcup T}=\left(\left.\mathrm{H}_{0}^{u}(x)\right|_{U \backslash\{u\}} ^{V}\right)_{T} .
\end{aligned}
$$

Definition 3.4 (Degenerate cubes, non-degenerate cubes). Let $x$ be an $S$-cube in a category $\mathcal{C}$.

(1) For $k \in S$, we say that $x$ is degenerate along the $k$-direction if $d_{T \sqcup\{k\}}^{x, k}$ is an isomorphism for any $T \in \mathcal{P}(S \backslash\{k\})$.

(2) We say that $x$ is non-degenerate if no boundary morphism of $x$ is an isomorphism.

Definition 3.5 (Total complexes). For an $n$-cube $x$ in an additive category $\mathcal{B}$, we associate the complex Tot $x$, called the total complex of $x$, defined as:

$$
(\operatorname{Tot} x)_{k}:=\bigoplus_{T \in \mathcal{P}((n]) \# T=k} x_{T}
$$

The boundary morphisms $d_{k}^{\operatorname{Tot} x}:(\operatorname{Tot} x)_{k} \rightarrow(\operatorname{Tot} x)_{k-1}$ are defined by

$$
(-1)^{\sum_{t=j+1}^{n} \chi_{T}(t)} d_{T}^{j}: x_{T} \rightarrow x_{T \backslash\{j\}}
$$

on its $x_{T}$ component to $x_{T \backslash\{j\}}$ component. Here $\chi_{T}$ is the characteristic function associated with $T$. (See 2.2). For a general finite set $S$, let us fix a bijection $\alpha:(n] \stackrel{\sim}{\rightarrow} S$. Then we can consider any $S$-cubes to be $n$-cubes by $\alpha$. Therefore we can define the total complex of an $S$-cube $x$ which is denoted by $\operatorname{Tot}_{\alpha} x$ or $\operatorname{simply} \operatorname{Tot} x$. Next moreover let us assume that $\mathcal{B}$ is an abelian category. We say that a morphism $f: x \rightarrow y$ between $S$-cubes in $\mathcal{B}$ is a quasi-isomorphism if $\operatorname{Tot} f: \operatorname{Tot} x \rightarrow \operatorname{Tot} y$ is a quasi-isomorphism. We denote the class of quasi-isomorphisms in $\mathbf{C u b}^{n} \mathcal{B}$ by ${ }^{\mathrm{t}} \mathrm{q}_{\mathbf{C u b}^{n}} \mathcal{B}$ or shortly tq.

Definition 3.6 (Spherical complex). Let $n$ be an integer. We say that a complex $z$ in an abelian category $\mathcal{A}$ is $n$-spherical if $\mathrm{H}_{k}(z)=0$ for any $k \neq n$.

From now on, in this section, let us assume that $S$ is a finite set and let $x$ be an $S$-cube in an abelian category $\mathcal{A}$.

Example 3.7 (Motivational example). Let $\left\{f_{s}\right\}_{s \in S}$ be a family of elements in $A$ which forms a regular sequence in any order. Then for any $1 \leqslant k \leqslant \# S$ and any distinct elements $s_{1}, \cdots, s_{k}$ in $S$, the boundary maps in the cube $\mathrm{H}_{0}^{s_{1}}\left(\cdots\left(\mathrm{H}_{0}^{s_{k}}\left(\operatorname{Typ}_{A}\left(\mathfrak{f}_{S}\right)\right)\right) \cdots\right)$ are injections where $\operatorname{Typ}_{A}\left(\mathfrak{f}_{S}\right)$ is the typical cube associated with $\left\{f_{s}\right\}_{s \in S}$ (See 2.7).

Definition 3.8 (Admissible cubes). If $\# S=1, x$ is said to be admissible if its boundary morphism is a monomorphism. Inductively, for $\# S>1, x$ is called admissible if its boundary morphisms are monomorphisms and if for any $k$ in $S, \mathrm{H}_{0}^{k}(x)$ is admissible. By convention, we say that $x$ is admissible if $S=\emptyset$. 
Remark 3.9. The name "admissible" comes from [29, p.331]. In [29, p.323 1.1.2], Waldhausen give a characterization of admissibility of squares. More precisely, for a square of monomorphisms in $\mathcal{A}$ below

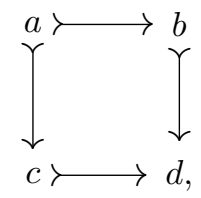

it is admissible if and only if the induced morphism $c \sqcup_{a} b \rightarrow d$ is a monomorphism.

Remark 3.10. Assume that $\# S \geqslant 2$. Then $x$ is admissible if and only if for any distinct elements $t, s_{1}, \cdots, s_{r}$ in $S(1 \leqslant r \leqslant \# S-1)$,

$$
\mathrm{H}_{1}^{t}(x) \quad \text { and } \quad \mathrm{H}_{1}^{t}\left(\mathrm{H}_{0}^{s_{1}}\left(\mathrm{H}_{0}^{s_{2}}\left(\cdots\left(\mathrm{H}_{0}^{s_{r}}(x)\right) \cdots\right)\right)\right)
$$

are trivial.

Lemma-Definition 3.11. For a pair of disjoint subsets $U$ and $V \in \mathcal{P}(S)$ such that $k:=\# U \geqslant 2$, let us assume that $\left.x\right|_{U} ^{V}$ is admissible. We denote the all distinct elements of $U$ by $i_{1}, \ldots, i_{k}$. Then we have the canonical isomorphism:

$$
\mathrm{H}_{0}^{i_{1}}\left(\mathrm{H}_{0}^{i_{2}}\left(\cdots\left(\mathrm{H}_{0}^{i_{k}}(x)\right) \cdots\right)\right)_{V} \stackrel{\sim}{\rightarrow} \mathrm{H}_{0}^{i_{\sigma(1)}}\left(\mathrm{H}_{0}^{i_{\sigma(2)}}\left(\cdots\left(\mathrm{H}_{0}^{i_{\sigma(k)}}(x)\right) \cdots\right)\right)_{V}
$$

where $\sigma$ is a bijection on $U$. In this case we put

$$
\mathrm{H}_{0}^{U}(x)_{V}:=\mathrm{H}_{0}^{i_{1}}\left(\mathrm{H}_{0}^{i_{2}}\left(\cdots\left(\mathrm{H}_{0}^{i_{k}}(x)\right) \cdots\right)\right)_{V} .
$$

We also put $\mathrm{H}_{0}^{\emptyset}(x):=x$. Notice that $\mathrm{H}_{0}^{T}(x)$ is an $S \backslash T$-cube for any $T \in \mathcal{P}(S)$.

Proof. We may assume that $U=S$ and $V=\emptyset$ by replacing $\left.x\right|_{U} ^{V}$ with $x$. Since every bijection on $S$ is expressed in compositions of substitutions of two elements, we shall just check the assertion for any substitution of two elements $\sigma$. Since for a pair of distinct elements $i, j \in S, x$ is considered to be a $\{i, j\}$-cube in $\mathbf{C u b}^{S \backslash\{i, j\}} \mathcal{A}$ by 2.4, we shall assume that $S=\{i, j\}$. In this case, by $3 \times 3$-lemma (See for example [30, Exercise 3.2.1]), we learn that $\mathrm{H}_{0}^{j}\left(\mathrm{H}_{0}^{i}(x)\right)_{\emptyset}$ and $\mathrm{H}_{0}^{i}\left(\mathrm{H}_{0}^{j}(x)\right)_{\emptyset}$ are canonically isomorphic to the object $y$ in the diagram below.

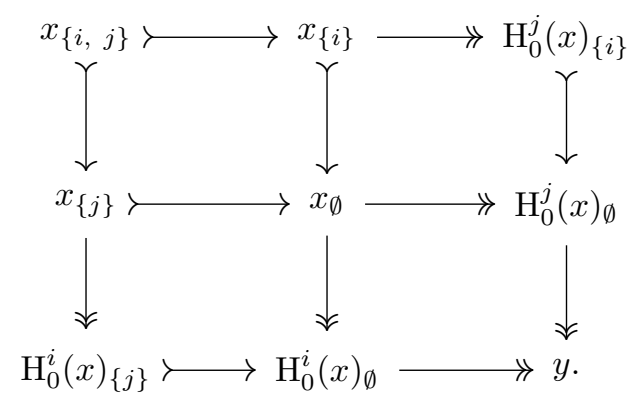

Lemma 3.12. Assume that $x$ is admissible. Then, for any pair of disjoint subsets $U$ and $V$ of $S,\left.x\right|_{U} ^{V}$ is admissible. In particular, all faces of $x$ are also admissible when $\# S \geqslant 1$. 
Proof. If $\# U \leqslant 1$, then the assertion is trivial. We assume that $\# U \geqslant 2$. For any distinct elements $u, s_{1}, \cdots, s_{r}$ of $U$, since $\mathrm{H}_{1}^{u}(x)$ and $\mathrm{H}_{1}^{u}\left(\mathrm{H}_{0}^{s_{1}}\left(\mathrm{H}_{0}^{s_{2}}\left(\cdots\left(\mathrm{H}_{0}^{s_{r}}(x)\right) \cdots\right)\right)\right)$ are trivial by assumption and $3.10, \mathrm{H}_{1}^{u}\left(\left.x\right|_{U} ^{V}\right)$ and $\mathrm{H}_{1}^{u}\left(\mathrm{H}_{0}^{s_{1}}\left(\mathrm{H}_{0}^{s_{2}}\left(\cdots\left(\mathrm{H}_{0}^{s_{r}}\left(\left.x\right|_{U} ^{V}\right)\right) \cdots\right)\right)\right)$ are also trivial by the equalities

$$
\begin{gathered}
\mathrm{H}_{1}^{u}\left(\left.x\right|_{U} ^{V}\right)=\left.\mathrm{H}_{1}^{u}(x)\right|_{U \backslash\{u\}} ^{V}, \\
\mathrm{H}_{1}^{u}\left(\mathrm{H}_{0}^{s_{1}}\left(\mathrm{H}_{0}^{s_{2}}\left(\cdots\left(\mathrm{H}_{0}^{s_{r}}\left(\left.x\right|_{U} ^{V}\right)\right) \cdots\right)\right)\right)=\left.\mathrm{H}_{1}^{u}\left(\mathrm{H}_{0}^{s_{1}}\left(\mathrm{H}_{0}^{s_{2}}\left(\cdots\left(\mathrm{H}_{0}^{s_{r}}(x)\right) \cdots\right)\right)\right)\right|_{U \backslash\left\{u, s_{1}, \cdots, s_{r}\right\}} ^{V}
\end{gathered}
$$

which come from 3.3. Hence $\left.x\right|_{U} ^{V}$ is admissible.

Proposition 3.13. Let us assume that $S$ is a non-empty set and all faces of $x$ are admissible. Then

(1) For any element $k$ in $S$, we have the following isomorphisms

$$
\omega_{k, S, x}^{p}: \mathrm{H}_{p}(\operatorname{Tot} x) \stackrel{\sim}{\rightarrow} \begin{cases}\mathrm{H}_{p}^{k}\left(\mathrm{H}_{0}^{S \backslash\{k\}}(x)\right) & \text { for } p=0,1 \\ 0 & \text { otherwise }\end{cases}
$$

which is functorial in the following sense: For any $S$-cube $y$ in $\mathcal{A}$ such that all faces are admissible and for any morphism $f: x \rightarrow y$ of $S$-cubes, the following diagram is commutative for $p=0,1$ :

$$
\begin{aligned}
& \mathrm{H}_{p} \operatorname{Tot} x \stackrel{\mathrm{H}_{p} \operatorname{Tot}(f)}{\longrightarrow} \mathrm{H}_{p} \operatorname{Tot} y \\
& \omega_{k, S, x}^{p} \downarrow 2 \\
& \mathrm{H}_{p}^{k} \mathrm{H}_{0}^{S \backslash\{k\}}(x) \underset{\mathrm{H}_{p}^{k}}{\longrightarrow} \mathrm{H}_{0}^{S \backslash\{k\}}(f) \mathrm{H}_{p}^{k} \mathrm{H}_{0}^{S \backslash\{k\}}(y) .
\end{aligned}
$$

(2) In particular, if $x$ is admissible, then we have the isomorphisms.

$$
\eta_{S, x}^{p}: \mathrm{H}_{p}(\operatorname{Tot} x) \stackrel{\sim}{\rightarrow} \begin{cases}\mathrm{H}_{0}^{S}(x) & \text { for } p=0 \\ 0 & \text { otherwise. }\end{cases}
$$

Proof. First we prove that assertion (1) implies assertion (2). Assume $x$ is admissible. Then all faces of $x$ and $\mathrm{H}_{0}^{S \backslash\{k\}}(x)$ for all $k \in S$ are admissible by 3.12 and the definition of admissibility. In particular $\mathrm{H}_{1}^{k} \mathrm{H}_{0}^{S \backslash\{k\}}(x)$ is trivial. Hence we obtain the result by (1).

Next we will prove assertion (1). We proceed by induction on the cardinality of $S$. Let us assume that the assertion is true for any $S \backslash\{k\}$-cubes in $\mathcal{A}$ which satisfy the assumption. For simplicity, we put $T=S \backslash\{k\}$ and $y_{1}=\left.x\right|_{T} ^{\{k\}}$ and $y_{0}=\left.x\right|_{T} ^{\emptyset}$ and $d:=d_{T}^{x, k}$. We regard $x$ as the $\{k\}$-cube $x=\left[y_{1} \stackrel{d}{\rightarrow} y_{0}\right]$ in $\mathbf{C u b}^{T} \mathcal{A}$. Since we have the isomorphisms Tot Cone $d \stackrel{\sim}{\rightarrow}$ Cone Tot $d \stackrel{\sim}{\rightarrow}$ Tot $x$, there is a distinguished triangle

$$
\text { Tot } y_{1} \stackrel{\text { Tot } d}{\rightarrow} \text { Tot } y_{0} \rightarrow \text { Tot } x \stackrel{+1}{\rightarrow}
$$

in the homotopy category of bounded complexes on $\mathcal{A}$. Here $\mathrm{H}_{p}$ Tot $y_{q}$ is trivial for any $p \geqslant 1$ and $q=0,1$ by the inductive assumption and assertion (2). Therefore the long exact sequence induced from the distinguished triangle (3) shows that $\mathrm{H}_{p} \operatorname{Tot} x$ 
is trivial for $p \geqslant 2$ and yields the top exact sequence in the commutative diagram below.

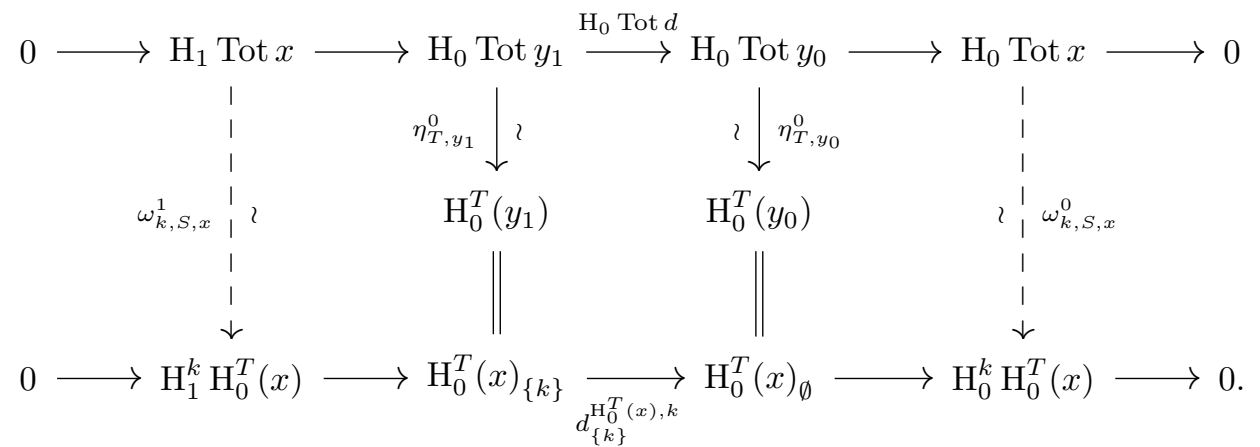

Then there are the isomorphisms $\omega_{k, S, x}^{p}: \mathrm{H}_{p}$ Tot $x \stackrel{\sim}{\rightarrow} \mathrm{H}_{p}^{k} \mathrm{H}_{0}^{T}(x)$ for $p=0,1$ which make the diagram above commutative. By construction, $\omega_{k, S, x}^{p}$ is functorial.

Corollary 3.14. For a pair of disjoint finite subsets $U, V$ of $S$, let us assume that $\left.x\right|_{U} ^{V}$ is admissible. Then we have the isomorphisms below:

$$
\mathrm{H}_{p}\left(\operatorname{Tot}\left(\left.x\right|_{U} ^{V}\right)\right) \stackrel{\sim}{\rightarrow} \begin{cases}\mathrm{H}_{0}^{U}(x)_{V} & \text { for } p=0 \\ 0 & \text { otherwise. }\end{cases}
$$

Proof. If $U=\emptyset$, we have the equality $\left.x\right|_{U} ^{V}=x_{V}=\mathrm{H}_{0}^{\emptyset}(x)_{V}$. Therefore the assertion is obvious. If $U \neq \emptyset$ applying 3.13 to $\left.x\right|_{U} ^{V}$ and noticing that the equality $\mathrm{H}_{0}^{U}\left(\left.x\right|_{U} ^{V}\right)_{\emptyset}=$ $\mathrm{H}_{0}^{U}(x)_{V}$, we get the result.

Corollary 3.15. The following conditions are equivalent:

(1) $x$ is admissible.

(2) Tot $x$ is 0 -spherical and all faces of $x$ are admissible.

Proof. Condition (1) implies condition (2) by 3.12 and 3.13 (2). Conversely let us assume that $x$ satisfies condition (2). We may assume $\# S \geqslant 1$. We prove that for any disjoint pair $V$ and $W \in \mathcal{P}(S) \backslash\{S\}$ and $k \in W$, the boundary morphism

$$
d_{W}^{\mathrm{H}_{0}^{V}(x), k}: \mathrm{H}_{0}^{V}(x)_{W} \rightarrow \mathrm{H}_{0}^{V}(x)_{W \backslash\{k\}}
$$

is a monomorphism. If $\#(S \backslash V) \geqq 2$, set $x^{\prime}$ equal to $\left.x\right|_{V \sqcup\{k\}} ^{W \backslash\{k\}}$ and then we can identify the boundary morphism above with the following morphism

$$
d_{\{k\}}^{\mathrm{H}_{0}\left(x^{\prime}\right), k}: \mathrm{H}_{0}\left(x^{\prime}\right)_{\{k\}} \rightarrow \mathrm{H}_{0}\left(x^{\prime}\right)_{\emptyset} .
$$

Therefore the assertion follows from admissibility of $x^{\prime}$. If $S \backslash V$ is the singleton $\{k\}$, then $W=\{k\}$ and by 3.13 , we have the isomorphisms $\mathrm{H}_{1}^{k}\left(\mathrm{H}_{0}^{S \backslash\{k\}}(x)\right) \stackrel{\sim}{\rightarrow} \mathrm{H}_{1}(\operatorname{Tot} x)=$ 0 . This means that we get the desired result.

Corollary 3.16 (Inductive characterization of admissibility). Assume that $S$ is a non-empty set. Then the following conditions are equivalent: 
(1) $x$ is admissible.

(2) For some $s \in S, x$ satisfies the following three conditions:

(i) $\left.x\right|_{S \backslash\{s\}} ^{\{s\}},\left.x\right|_{S \backslash\{s\}} ^{\emptyset}$ are admissible.

(ii) $d_{T \sqcup\{s\}}^{x, s}$ is a monomorphism for any $T \in \mathcal{P}(S \backslash\{s\})$.

(iii) $\mathrm{H}_{0}^{s}(x)$ is admissible.

(3) For any $s \in S, x$ satisfies the three conditions (i), (ii) and (iii) in (2).

Proof. We can easily check that condition (1) implies condition (3) and condition (3) implies condition (2). We need only prove that condition (2) implies condition (1). We prove this assertion by induction on $\# S$. If $\# S=1$, the assertion is trivial. For $\# S>1$, we will prove that

(a) all faces of $x$ are admissible, and

(b) Tot $x$ is 0 -spherical.

Proof for assertion (a): We prove that for any $k \in S$, the faces $\left.x\right|_{S \backslash\{k\}} ^{\{k\}}$ and $\left.x\right|_{S \backslash\{k\}} ^{\emptyset}$ are admissible. If $k=s$, it is just condition (i). If $k \neq s$, then they satisfy conditions (i), (ii) and (iii) and therefore by the inductive hypothesis, they are admissible.

Proof for assertion (b): Fix an element $t \in S \backslash\{s\}$. Since we have the isomorphism

$$
\mathrm{H}_{0}^{S \backslash\{t\}}(x) \stackrel{\sim}{\rightarrow} \mathrm{H}_{0}^{S \backslash\{s, t\}}\left(\mathrm{H}_{0}^{s}(x)\right),
$$

we learn that $\mathrm{H}_{0}^{S \backslash\{t\}}(x)$ is admissible by condition (iii). In particular, $\mathrm{H}_{1}^{t}\left(\mathrm{H}_{0}^{S \backslash\{t\}}(x)\right)$ is trivial. On the other hand, by 3.13 , we have the isomorphism

$$
\mathrm{H}_{p}(\operatorname{Tot} x) \stackrel{\sim}{\rightarrow} \begin{cases}\mathrm{H}_{p}^{t}\left(\mathrm{H}_{0}^{S \backslash\{t\}}(x)\right) & \text { for } p=0,1 \\ 0 & \text { otherwise. }\end{cases}
$$

Therefore we notice that Tot $x$ is 0 -spherical. Hence by $3.15, x$ is admissible.

Corollary 3.17. For a subset $T \subset S$, if the following two conditions are verified, then $x$ is admissible:

(1) $x$ is degenerate along the $k$-direction for any $k \in T$.

(2) $\left.x\right|_{S \backslash T} ^{\emptyset}$ is admissible.

Proof. Since for any element in $t \in T$, we have the equality

$$
\left.\left(\left.x\right|_{S \backslash(T \backslash\{t\})} ^{\emptyset}\right)\right|_{S \backslash T} ^{\emptyset}=\left.x\right|_{S \backslash T} ^{\emptyset},
$$

by the induction of $\# T$, we shall assume that $T$ is the singleton $T=\{k\}$. In this case, $x$ satisfies conditions (i), (ii) and (iii) in 3.16 (2) for $s=k$. Therefore $x$ is admissible.

Definition 3.18 (Multi semi-direct products). Let $\mathfrak{F}=\left\{\mathcal{F}_{T}\right\}_{T \in \mathcal{P}(S)}$ be a family of full subcategories of $\mathcal{A}$. Then

(1) We define $\ltimes \mathfrak{F}=\underset{T \in \mathcal{P}(S)}{\ltimes} \underset{\mathcal{F}_{T}}{ }$ the multi semi-direct product of the family $\mathfrak{F}$ as follows. $\underset{T \in \mathcal{P}(S)}{\ltimes} \mathcal{F}_{T}$ is the full subcategory of $\mathbf{C u b}^{S}\left(\mathcal{F}_{\emptyset}\right)$ consisting of those cubes $x$ such that $x$ is admissible and each vertex of $\mathrm{H}_{0}^{T}(x)$ is in $\mathcal{F}_{T}$ for any $T \in \mathcal{P}(S)$. If $S$ is a singleton, then $\underset{T \in \mathcal{P}(S)}{\ltimes} \mathcal{F}_{T}$ is just the semi-direct product $\mathcal{F}_{S} \ltimes \mathcal{F}_{\emptyset}$ in 2.9 . 
(2) Suppose in addition that $\mathcal{F}_{S}$ has a class of weak equivalences $w=w\left(\mathcal{F}_{S}\right)$. Then a morphism $f: x \rightarrow y$ in $\ltimes \mathfrak{F}$ is said to be a total weak equivalences if $\mathrm{H}_{0}^{S}(f)$ is in $w$. We denote the class of total weak equivalences in $\ltimes \mathfrak{F}$ by $t w\left(\underset{T \in \mathcal{P}(S)}{\ltimes} \mathcal{F}_{T}\right)$ or simply $t w$.

Proposition 3.19. Let $\mathfrak{F}=\left\{\mathcal{F}_{T}\right\}_{T \in \mathcal{P}(S)}$ be a family of full subcategories of $\mathcal{A}$. Then

(1) For each $s \in S$, we have the equality

$$
\underset{T \in \mathcal{P}(S)}{\ltimes} \mathcal{F}_{T}=\left(\underset{T \in \mathcal{P}(S \backslash\{s\})}{\stackrel{\ltimes}{\ltimes}} \mathcal{F}_{T \sqcup\{s\}}\right) \ltimes\left(\underset{T \in \mathcal{P}(S \backslash\{s\})}{\stackrel{\times}{\times}} \mathcal{F}_{T}\right) .
$$

(2) In the equality in (1), the class of quasi-isomorphisms in $\underset{T \in \mathcal{P}(S)}{\ltimes} \mathcal{F}_{T}$ is equal to the class of weak equivalences induced from the class of quasi-isomorphisms in $\underset{T \in \mathcal{P}(S \backslash\{s\})}{\ltimes} \mathcal{F}_{T \sqcup\{s\}}$ as in 2.11. Namely we have the equality

$$
t w\left(\underset{T \in \mathcal{P}(S)}{\ltimes} \mathcal{F}_{T}\right)=t\left(t w\left(\underset{T \in \mathcal{P}(S \backslash\{s\})}{\ltimes} \mathcal{F}_{T \sqcup\{s\}}\right)\right) .
$$

(3) In the situation (2), we have the equality

$$
\left(\underset{T \in \mathcal{P}(S)}{\ltimes} \mathcal{F}_{T}\right)^{t w}=\left(\underset{T \in \mathcal{P}(S \backslash\{s\})}{\stackrel{\ltimes}{\ltimes}} \mathcal{F}_{T \sqcup\{s\}}\right)^{t w} \ltimes\left(\underset{T \in \mathcal{P}(S \backslash\{s\})}{\ltimes} \mathcal{F}_{T}\right) .
$$

Proof. Proof of assertion (1): Let us fix an element $s \in S$. For simplicity, we put

$$
\mathcal{G}=\left(\underset{T \in \mathcal{P}(S \backslash\{s\})}{\ltimes} \mathcal{F}_{T \sqcup\{s\}}\right) \ltimes\left(\underset{T \in \mathcal{P}(S \backslash\{s\})}{\ltimes} \mathcal{F}_{T}\right) .
$$

Let $x$ be an object in $\ltimes \mathcal{F}$. To prove that $x$ is in $\mathcal{G}$, we need to check the following two assertions:

(a) $\left.x\right|_{S \backslash\{s\}} ^{\{s\}},\left.x\right|_{S \backslash\{s\}} ^{\emptyset}$ are in $\underset{T \in \mathcal{P}(S \backslash\{s\})}{\ltimes} \mathcal{F}_{T}$.

(b) $\mathrm{H}_{0}^{s}(x)$ is in $\underset{T \in \mathcal{P}(\stackrel{S}{\ltimes} \backslash\{s\})}{\times} \mathcal{F}_{T \sqcup\{s\}}$.

We put $W=\emptyset$ or $W=\{s\}$. First let us notice that $\left.x\right|_{S \backslash\{s\}} ^{W}$ and $\mathrm{H}_{0}^{s}(x)$ are admissible by admissibility of $x$. For each $T \in \mathcal{P}(S \backslash\{s\})$ and $V \in \mathcal{P}(S \backslash(T \sqcup\{s\}))$, we have the equalities

$$
\begin{gathered}
\mathrm{H}_{0}^{T}\left(\mathrm{H}_{0}^{s}(x)\right)_{V}=\mathrm{H}_{0}^{T \sqcup\{s\}}(x)_{V} \text { and } \\
\mathrm{H}_{0}^{T}\left(\left.x\right|_{S \backslash\{s\}} ^{W}\right)_{V}=\mathrm{H}_{0}^{T}(x)_{V \sqcup W} .
\end{gathered}
$$

Therefore both objects above are in $\mathcal{F}_{T \sqcup W}$. Hence we get assertions (a) and (b) and we learn that $x$ is in $\mathcal{G}$. Conversely next let $x$ be an object in $\mathcal{G}$. Since $x$ satisfies conditions (i), (ii) and (iii) in by $3.16(2), x$ is admissible. Moreover by the equalities (4) and (5) above, we learn that $\mathrm{H}_{0}^{T}(x)_{V}$ is in $\mathcal{F}_{T}$ for any disjoint pair of subsets $T$, $V \in \mathcal{P}(S)$. Therefore $x$ is in $\ltimes \mathfrak{F}$. 
Proof of assertion (2): By 3.14, a morphism $f: x \rightarrow y$ in

$$
\underset{T \in \mathcal{P}(S)}{\ltimes} \mathcal{F}_{T}
$$

is a quasi-isomorphism if and only if $\mathrm{H}_{0}(\operatorname{Tot} f): \mathrm{H}_{0}(\operatorname{Tot} x) \rightarrow \mathrm{H}_{0}$ (Tot $\left.y\right)$ is an isomorphism. Since $\mathrm{H}_{0}(\operatorname{Tot} z)=\mathrm{H}_{0}\left(\operatorname{Tot} \mathrm{H}_{0}^{s}(z)\right)(z=x, y)$, this condition is equivalent to the assertion that the induced morphism $\mathrm{H}_{0}^{s}(f): \mathrm{H}_{0}^{s}(x) \rightarrow \mathrm{H}_{0}^{s}(y)$ is a quasi-isomorphism. Hence we get the result.

Proof of assertion (3): By virtue of the equality in (1), we may assume that $S$ is a singleton. Namely $\# S=1$. For simplicity we put $\mathcal{E}=\mathcal{F}_{\emptyset}$ and $\mathcal{F}=\mathcal{F}_{S}$. For any object $x$ in $\mathcal{F} \ltimes \mathcal{E}$, the canonical morphism $0 \rightarrow x$ is in $t w$ if and only if the canonical morphism $0 \rightarrow \mathrm{H}_{0}(x)$ is in $w$ and the last assertion is equivalent to $\mathrm{H}_{0}(x)$ being in $\mathcal{F}^{w}$. Hence we get the desired result.

Corollary 3.20. Let $\mathfrak{E}=\left\{\mathcal{E}_{T}\right\}_{T \in \mathcal{P}(S)}$ and $\mathfrak{F}=\left\{\mathcal{F}_{T}\right\}_{T \in \mathcal{P}(S)}$ be families of subcategories of $\mathcal{A}$ such that for each $T \in \mathcal{P}(S), \mathcal{F}_{T} \hookrightarrow \mathcal{E}_{T}$ are strict exact subcategories of $\mathcal{A}$ and the inclusion functor $\mathcal{E}_{T} \hookrightarrow \mathcal{A}$ is closed under extensions. Then

(1) $\ltimes \mathfrak{E}$ is closed under extensions in $\mathbf{C u b}^{S} \mathcal{A}$. In particular $\ltimes \mathfrak{E}$ naturally becomes an exact category.

(2) If $\mathcal{F}_{T} \hookrightarrow \mathcal{E}_{T}$ is closed under extensions (resp. taking admissible sub- and quotient objects, taking kernels of admissible epimorphisms, taking finite direct sum) for any $T \in \mathcal{P}(S)$, then the inclusion functor $\ltimes \mathfrak{F} \hookrightarrow \ltimes \mathfrak{E}$ is also closed under extensions (resp. taking admissible sub-and quotient objects, taking kernels of admissible epimorphisms, taking finite direct sum).

(3) Assume that the following two conditions hold:

(i) For any pair of subsets $T \subset U$ in $S, \mathcal{E}_{U}$ is full subcategory of $\mathcal{E}_{T}$.

(ii) For any subset $T$ in $S$, every object in $\mathcal{F}_{T}$ is a projective object in $\mathcal{E}_{T}$. Then every object in $\ltimes \mathfrak{F}$ is a projective object in $\ltimes \mathfrak{E}$.

Proof. Utilizing the inductive description of $\ltimes \mathfrak{E}$ and $\ltimes \mathfrak{F}$ as in 3.19 (1), we get the results by induction and $2.14,2.15,2.16$ and 2.17 .

Remark 3.21. Let $\mathfrak{F}=\left\{\mathcal{F}_{T}\right\}_{T \in \mathcal{P}(S)}$ be a family of strict exact subcategories of $\mathcal{A}$. Assume that for any disjoint decomposition $S=U \sqcup V, \underset{T \in \mathcal{P}(U)}{\stackrel{\sim}{\ltimes}} \mathcal{F}_{T \sqcup V}$ is a strict exact subcategory of $\mathbf{C u b}^{U}(\mathcal{A})$.

(1) Since boundary morphisms of admissible cubes are monomorphisms, for each $s \in S$, the functor

$$
\mathrm{H}_{0}^{s}: \underset{T \in \mathcal{P}(S)}{\ltimes} \mathcal{F}_{T} \rightarrow \underset{T \in \mathcal{P}(S \backslash\{s\})}{\ltimes} \mathcal{F}_{T \sqcup\{s\}}
$$

is exact. Moreover by induction on the number of elements, we learn that for any $W \in \mathcal{P}(S)$,

$$
\mathrm{H}_{0}^{W}: \underset{U \in \mathcal{P}(S)}{\ltimes} \mathcal{F}_{U} \rightarrow \underset{T \in \mathcal{P}(S \backslash W)}{\ltimes} \underset{\mathcal{F}_{W}}{\ltimes}
$$

is also an exact functor. 
(2) In particular, since for the functors $\mathrm{H}_{0}^{S}, \mathrm{H}_{0}$ Tot: $\ltimes \mathfrak{F} \rightarrow \mathcal{F}_{S}$, we have the isomorphism $\mathrm{H}_{0}^{S} \stackrel{\sim}{\rightarrow} \mathrm{H}_{0}$ Tot by $3.13(2), \mathrm{H}_{0}$ Tot is also an exact functor from $\ltimes \mathfrak{F}$ to $\mathcal{F}_{S}$.

\section{Koszul cubes}

In this section, we define Koszul cubes in 4.8 and relate them to semi-direct products of exact categories of pure weight modules as in 4.20. The pivot of the theory is Theorem 4.15 which says that the total complexes associated with free non-degenerate Koszul cubes are 0 -spherical. Let us commence defining and recalling elementary facts about regular sequences. Let $R$ (resp., $A$ ) be a commutative ring with 1 (resp., commutative noetherian ring with 1 ).

By an $A$-sequence we mean an $A$-regular sequence $f_{1}, \cdots, f_{q}$ such that any permutation of the $f_{j}$ is also an $A$-regular sequence.

Example 4.1. We enumerate fundamental properties of $A$-sequences from $[\mathbf{1 9}, \S 16]$.

(1) For any $A$-regular sequence $f_{1}, \cdots, f_{q}$ and a prime ideal $\mathfrak{p}$ in $A$ such that $f_{i} \in \mathfrak{p}$ for any $i \in(q], f_{1}, \cdots, f_{q}$ is an $A_{\mathfrak{p}}$-regular sequence in $A_{\mathfrak{p}}$. In particular if $f_{1}, \cdots, f_{q}$ is an $A$-sequence, then it is also an $A_{\mathfrak{p}}$-sequence in $A_{\mathfrak{p}}$.

(2) For any $A$-regular sequence $f_{1}, \cdots, f_{q}$ and positive integers $\mu_{1}, \cdots, \mu_{q}, f_{1}^{\mu_{1}}, \cdots$, $f_{q}^{\mu_{q}}$ is again an $A$-regular sequence. In particular if $f_{1}, \cdots, f_{q}$ is an $A$-sequence, then $f_{1}^{\mu_{1}}, \cdots, f_{q}^{\mu_{q}}$ is also an $A$-sequence.

(3) Any $A$-regular sequence contained in the Jacobson radical of $A$ is automatically an $A$-sequence.

The following lemma might be well-known. But I do not know a reference and we give a proof in minute detail:

Lemma 4.2. Let $R$ be a commutative ring with unit and $f_{1}, \cdots, f_{n}, g_{1}, \cdots, g_{n}$ elements in $R$. We put $h_{i}=f_{i} g_{i}$ for each $1 \leqslant i \leqslant n$ and assume that each $f_{i}$ is not a unit and the sequence $h_{1}, \cdots, h_{n}$ is an $R$-sequence. Then $f_{1}, \cdots, f_{n}$ is also an $R$-sequence.

Proof. If $n=1, f_{1}$ is actually a non zero divisor. For $n>1$, by induction on $n$, we shall only check that if for some elements $x$ and $y_{i}^{(0)}$ in $R(1 \leqslant i \leqslant n)$, we have the equality

$$
f_{n} x=\sum_{i=1}^{n-1} f_{i} y_{i}^{(0)},
$$

then we have the equality $x=\sum_{i=1}^{n-1} f_{i} z_{i}$ for some elements $z_{i}$ in $R$. Multiplying the equality (6) by $g=\prod_{i=1}^{n} g_{i}$, we get the equality

$$
h_{n}\left(\prod_{i=1}^{n-1} g_{i}\right) x=\sum_{i=1}^{n-1} h_{i}\left(\frac{g}{g_{i}} y_{i}^{(0)}\right) .
$$

Since the sequence $h_{1}, \cdots, h_{n}$ is an $R$-sequence, there are elements $y_{1}^{(1)}, \cdots, y_{n-1}^{(1)}$ in 
$R$ such that

$$
\left(\prod_{i=1}^{n-1} g_{i}\right) x=\sum_{i=1}^{n-1} h_{i} y_{i}^{(1)} .
$$

Now let us define the polynomials $\varphi_{x, i}\left(Y_{1}, \cdots, Y_{n-1}\right)$ in $R\left[Y_{1}, \cdots, Y_{n-1}\right](1 \leqslant i \leqslant n)$ by the formula

$$
\varphi_{x, i}\left(Y_{1}, Y_{2}, \cdots, Y_{n-1}\right)= \begin{cases}g_{1}\left(x-f_{1} Y_{1}\right) & \text { if } i=1 \text { and } n=2 \\
g_{1}\left\{\left(\begin{array}{l}
n-1 \\
\prod_{j=2} g_{j}
\end{array}\right) x-f_{1} Y_{1}\right\}-\sum_{j=2}^{n-1} h_{j} Y_{j} & \text { if } i=1 \text { and } n \geqslant 3 \\
g_{i}\left\{\left(\prod_{j=i+1}^{n-1} g_{j}\right) x-f_{i} Y_{i}\right\}-\left(\sum_{j=1}^{i-1} f_{j} Y_{j}+\sum_{j=i+1}^{n-1} h_{j} Y_{j}\right) & \text { if } 2 \leqslant i \leqslant n-2 \\
g_{n-1}\left(x-f_{n-1} Y_{n-1}\right)-\sum_{j=1}^{n-2} f_{j} Y_{j} & \text { if } i=n-1 \text { and } n \geqslant 3 \\
x-\sum_{j=1}^{n-1} f_{j} Y_{j} & \text { if } i=n .\end{cases}
$$

Claim. For $1 \leqslant i \leqslant n-1$, if $\varphi_{x, i}=0$ has a solution in $R$, then the equation $\varphi_{x, i+1}=0$ also has a solution in $R$.

Proof of claim. Let a system $Y_{j}=y_{j}(1 \leqslant j \leqslant n-1)$ be a solution of $\varphi_{x, i}=0$. If $g_{i}$ is a unit, then the system

$$
Y_{j}= \begin{cases}g_{i}^{-1} y_{j} & \text { if } j \neq i \\ y_{i} & \text { if } j=i\end{cases}
$$

is a solution of $\varphi_{x, i+1}=0$. If $g_{i}$ is not a unit, then by inductive hypothesis, the sequence

$$
\begin{cases}g_{1} & \text { (if } i=1 \text { and } n=2) \\ h_{2}, \cdots, h_{n-1}, g_{1} & \text { (if } i=1 \text { and } n \geqslant 3) \\ f_{1}, f_{2}, \cdots, f_{i-1}, h_{i+1}, \cdots, h_{n-1}, g_{i} & \text { (if } 2 \leqslant i \leqslant n-2 \text { and } n \geqslant 4) \\ f_{1}, \cdots, f_{n-2}, g_{n-1} & \text { (if } i=n-1 \text { and } n \geqslant 3)\end{cases}
$$

is an $R$-sequence. Therefore we have elements $z_{j}$ in $R(1 \leqslant j \leqslant n-1, j \neq i)$ such that the system

$$
Y_{j}= \begin{cases}z_{j} & \text { if } j \neq i \\ y_{i} & \text { if } j=i\end{cases}
$$

is a solution of $\varphi_{x, i+1}=0$.

Since the system $Y_{j}=y_{j}^{(1)}(1 \leqslant j \leqslant n-1)$ is a solution of $\varphi_{x, 1}=0$, by induction on $i$, finally we have a solution of $\varphi_{x, n}=0$ and this is just the desired result.

The following assertion is a very basic fact about regular sequences and useful to handle Koszul cubes:

Lemma 4.3. For a non-zero divisor $f$ in $R$, an $R$-sequence $g, h$ in $R$, we have the equalities

$$
\begin{gathered}
R_{f}^{\times} \cap R=\left\{r \in R ; f^{m}=r u \text { for some } m \in \mathbb{N}, u \in R\right\} \quad \text { and } \\
R_{g}^{\times} \cap R_{h}^{\times}=R^{\times}
\end{gathered}
$$

in the total quotient ring of $R$. 
Proof. We will give a proof of the second equality above. An element $u \in R_{g} \cap R_{h}$ is of the form $u=\frac{x}{g^{n}}=\frac{y}{h^{m}}$ for some positive integers $n$ and $m$. Then we have the equality $h^{m} x=g^{n} y$. Since the sequence $g^{n}, h^{m}$ is an $R$-sequence by 4.1 (2), there is an element $z$ in $R$ such that $x=g^{n} z$ and therefore $u=\frac{z}{1}$. Hence we have $R_{g} \cap R_{h}=R$ in the total quotient ring of $R$ and it implies the desired equality $R_{g}^{\times} \cap R_{h}^{\times}=\left(R_{g} \cap R_{h}\right)^{\times}=$ $R^{\times}$.

Next we will make ready for the general jargons of cubes in the category of Modules over a commutative ring with unit $R$.

Definition 4.4 (Free, projective, finitely generated cubes). We say that a cube $x$ in the category of $R$-Modules is free (resp. projective, finitely generated) if all vertexes of $x$ are free (resp. projective, finitely generated) $R$-Modules.

Definition 4.5 (Localization of cubes in the category of Modules). For any $S$-cube $x$ in the category of $R$-Modules and a multiplicative subset $\mathfrak{S} \subset R, \mathfrak{S}^{-1} x$ will denote the $S$-cube in the category of $\mathfrak{S}^{-1} R$-Modules defined by $\left(\mathfrak{S}^{-1} x\right)_{T}:=\mathfrak{S}^{-1}\left(x_{T}\right)$ for any $T \in \mathcal{P}(S)$. If $\mathfrak{S}=\left\{f^{n}\right\}_{n \geqslant 0}$ (reps. $A \backslash \mathfrak{p}$ ) for some element $f \in R$ (resp. a prime ideal $\mathfrak{p}$ in $R$ ), we write $x_{f}$ (resp. $x_{\mathfrak{p}}$ ) for $\mathfrak{S}^{-1} x$.

Definition 4.6. Let the letter $p$ be a natural number or $\infty$ and $I$ an ideal of $A$. Let $\mathcal{M}_{A}^{I}(p)$ denote the category of finitely generated $A$-modules $M$ such that $\operatorname{Projdim}_{A} M \leqslant p$ and $\operatorname{Supp} M \subset V(I)$. We write $\mathcal{M}_{A}^{I}$ for $\mathcal{M}_{A}^{I}(\infty)$. Since this category is closed under extensions in $\mathcal{M}_{A}$, it can be considered to be an exact category in the natural way. Notice that if $I$ is the zero ideal of $A$, then $\mathcal{M}_{A}^{I}(0)$ is just the category $\mathcal{P}_{A}$.

Remark 4.7. In [15], a pseudo-coherent $\mathcal{O}_{X}$-Module on a scheme $X$ is said to be of Thomason-Trobaugh weight $q$ if it is supported on a regular closed immersion $Y \hookrightarrow X$ of codimension $q$ and if it is of Tor-dimension $\leqslant q$. The following are equivalent for any finitely generated $A$-module $M$ and any ideal $I$ which is generated by an $A$-regular sequence $f_{1}, \cdots, f_{r}$ :

(1) The quasi-coherent $\mathcal{O}_{\operatorname{Spec} A}$-module associated with $M$ is of Thomason-Trobaugh weight $r$ supported on $V(I)$.

(2) $M$ is in $\mathcal{M}_{A}^{I}(r)$.

This is a consequence of the two facts that $\operatorname{Tordim}_{A} M=\operatorname{Projdim}_{A} M$ (see [30, Proposition 4.1.5]) and that $\operatorname{Spec} A / I \rightarrow \operatorname{Spec} A$ is a regular closed immersion of codimension $r$.

In this section, from now on, assume that $S$ is a finite set and let $\left\{f_{s}\right\}_{s \in S}$ be an $A$-sequence and let us fix $0 \leqslant p \leqslant \infty$.

Definition 4.8 (Koszul cube). A Koszul cube $x$ associated with $\left\{f_{s}\right\}_{s \in S}$ is an $S$ cube in the category of finitely generated projective $A$-modules $\mathcal{P}_{A}$ such that for each subset $T$ of $S$ and $k$ in $T, d_{T}^{k}$ is an injection and Coker $d_{T}^{k}$ is in $\mathcal{M}_{A}^{f_{k} A}(1)$. We denote the full subcategory of $\mathbf{C u b}^{S} \mathcal{P}_{A}$ consisting of those Koszul cubes associated with $\left\{f_{s}\right\}_{s \in S}$ by $\operatorname{Kos}_{A}^{\mathfrak{f}_{S}}$. Notice that if $S=\emptyset$, then $\operatorname{Kos}_{A}^{\mathfrak{f}_{S}}$ is just the category $\mathcal{P}_{A}$. 
Example 4.9. The typical cube associated with $\left\{f_{s}\right\}_{s \in S}$ in 2.7 is obviously a nondegenerate free Koszul cube associated with $\left\{f_{s}\right\}_{s \in S}$.

By 4.1 (1), we can get the following lemma easily:

Lemma 4.10 (Localization of Koszul cubes). Let $x$ be a Koszul cube associated with an A-sequence $\left\{f_{s}\right\}_{s \in S}$ and $\mathfrak{p}$ a prime ideal in $A$. We put $T:=\left\{t \in S ; f_{t} \in \mathfrak{p}\right\}$. Then

(1) $x_{\mathfrak{p}}$ is degenerate along the $t$-direction for any $t \in S \backslash T$.

(2) $\left.x_{\mathfrak{p}}\right|_{T} ^{\emptyset}$ is a Koszul cube associated with the $A_{\mathfrak{p}}$-sequence $\left\{f_{t}\right\}_{t \in T}$.

Lemma-Definition 4.11 (Determinant of free Koszul cubes). Let $x$ be a free Koszul $S$-cube associated with $\left\{f_{s}\right\}_{s \in S}$. Then

(1) All vertexes of $x$ have same rank.

(2) By virtue of (1), if we fix bases $\alpha$ of all vertexes of $x$, a boundary map $d_{T}^{k, x}$ of $x$ is represented by a square matrix $D_{T}^{k, x}$ and we write $\operatorname{det}_{\alpha} d_{T}^{k, x}$ or simply $\operatorname{det} d_{T}^{k, x}$ for $\operatorname{det} D_{T}^{k, x}$. For each $T \in \mathcal{P}(S), k \in T$, there is a unit element $u_{T, k}$ such that $\operatorname{det} d_{S}^{x, k}=u_{T, k} \times \operatorname{det} d_{T}^{x, k}$. We call the family $\left\{\operatorname{det} d_{S}^{x, k}\right\}_{k \in S}$ consisting of elements in $A$ determinant of $x$ (with respect to the bases $\alpha$ ) and denote it by $\operatorname{det}_{\alpha} x$ or simply det $x$. If we change bases of $x$ into others, then $\operatorname{det} x$ is changing up to units.

Proof. First we prove assertion (1). For any $T \in \mathcal{P}(S), k \in T$, since Coker $d_{T}^{x, k}$ is in $\mathcal{M}_{A}^{f_{k} A}(1)$, we learn that $d_{T}^{x, k}$ induces the isomorphism $\left(x_{T}\right)_{f_{k}} \stackrel{\sim}{\rightarrow}\left(x_{T \backslash\{k\}}\right)_{f_{k}}$. This isomorphism implies the equality $\operatorname{rank} x_{T}=\operatorname{rank} x_{T \backslash\{k\}}$. Hence we get the result. Next we prove assertion (2). We need only check that for any $T \in \mathcal{P}(S)$ and a pair of distinct elements $k, k^{\prime} \in T$, there is a unit element $v_{T, k, k^{\prime}}$ in $A$ such that $\operatorname{det} d_{T}^{x, k}=v_{T, k, k^{\prime}} \times \operatorname{det} d_{T \backslash\left\{k^{\prime}\right\}}^{x, k}$. Since in the total quotient ring of $A$, we have the equality $v_{T, k, k^{\prime}}:=\frac{\operatorname{det} d_{T}^{x, k}}{\operatorname{det} d_{T \backslash\left\{k^{\prime}\right\}}^{x, k}}=\frac{\operatorname{det} d_{T}^{x, k^{\prime}}}{\operatorname{det} d_{T \backslash\{k\}}^{x, k^{\prime}}}$, the element $v_{T, k, k^{\prime}}$ is in $A_{f_{k}}^{\times} \cap A_{f_{k^{\prime}}}^{\times}$. Hence by 4.3 , we learn that $v_{T, k, k^{\prime}}$ is the desired element.

Corollary-Definition 4.12 (Non-degenerate part of Koszul cubes). Let $x$ be a Koszul $S$-cube. If for some $T \in \mathcal{P}(S), t \in T, d_{T}^{x, t}$ is an isomorphism, then $x$ is degenerate along the $t$-direction. We put

$$
N_{x}:=\{s \in S ; x \text { is not degenerate along the } s \text {-direction. }\}
$$

and call $x_{\text {nondeg }}:=\left.x\right|_{N_{x}} ^{\emptyset}$ the non-degenerate part of $x$.

Proof. We need to check that for any $U \in \mathcal{P}(S)$ such that $t \in U, d_{U}^{x, t}$ is an isomorphism. Let us notice that the property of isomorphisms between modules is a local property. We fix a prime ideal $\mathfrak{p}$ in $A$ and put $V:=\left\{s \in S ; f_{s} \in \mathfrak{p}\right\}$. By 4.10 and replacing $A, x$ with $A_{\mathfrak{p}},\left.\left(x_{\mathfrak{p}}\right)\right|_{V}$, respectively, we shall assume that $A$ is local and $x$ is free Koszul cube. Then by $4.11(2), \operatorname{det} d_{T}^{x, t}$ is equal to $\operatorname{det} d_{U}^{x, t}$ up to a unit element. Hence we get the result. 
Lemma 4.13. Let $\phi: R^{\oplus n} \rightarrow R^{\oplus n}$ be a homomorphism of $R$-modules and assume that Coker $\phi$ is annihilated by a non-zero divisor $g \in R$. Then

(1) There exists an element $b \in R$ and a non-negative integer $m$ such that we have the equality $\operatorname{det} \phi \times b=g^{m}$.

(2) $\operatorname{det} \phi$ is a non-zero divisor in $R$.

(3) $\phi$ is an injection.

Proof. Localizing by $g$, we get the surjection $R_{g}^{\oplus n} \stackrel{\phi_{g}}{\rightarrow} R_{g}^{\oplus n}$. Since in general, a surjective homomorphisms between finitely generated free modules with same ranks are isomorphisms, it turns out that $\operatorname{det} \phi_{g}$ is in $R_{g}^{\times}$. Therefore we get assertion (1) by 4.3. Since $g$ is a non-zero divisor, $\operatorname{det} \phi$ is also a non-zero divisor. Hence we get assertion (2). Let $\phi^{*}$ be the adjugate of $\phi$, namely $\phi^{*}: R^{\oplus n} \rightarrow R^{\oplus n}$ is an $R$-module homomorphism such that $\phi^{*} \phi=\operatorname{det} \phi \operatorname{id}_{R^{\oplus n}}$. Since $\operatorname{det} \phi \operatorname{id}_{R^{\oplus n}}$ is an injection, we conclude that $\phi$ is also an injection.

Recall the definition of non-degenerate cubes from 3.4.

Proposition 4.14. For any non-degenerate free Koszul $S$-cube $x$, $\operatorname{det} x$ is an $A$ sequence.

Proof. Since $x$ is non-degenerate, for each $s \in S$, $\operatorname{det} d_{S}^{x, s}$ is not a unit element. By 4.13, there are a family of positive integers $\left\{m_{s}\right\}_{s \in S}$ such that $\operatorname{det} d_{S}^{x, s}$ is a divisor of $f_{s}^{m_{s}}$ for each $s \in S$. Therefore by 4.1 (2) and 4.2, $\operatorname{det} x$ forms an $A$-sequence.

Theorem 4.15. For any non-degenerate free Koszul $S$-cube $x$, Tot $x$ is 0 -spherical.

To prove the theorem, we use the Buchsbaum-Eisenbud Theorem 4.18 below.

Definition 4.16 (Fitting ideal). Let $U$ be an $m \times n$ matrix over $A$, where $m$ and $n$ are positive integers. For $t$ in $(\min (m, n)]$ we then denote by $I_{t}(U)$ the ideal generated by the $t$-minors of $U$, that is, the determinant of $t \times t$ sub-matrices of $U$.

For an $A$-module homomorphism $\phi: M \rightarrow N$ between free $A$-modules of finite rank, let us choose a matrix representation $U$ with respect to bases of $M$ and $N$. One can easily prove that the ideal $I_{t}(U)$ only depends on $\phi$. Therefore we put $I_{t}(\phi):=I_{t}(U)$ and call it the $t$-th Fitting ideal associated with $\phi$.

Definition 4.17 (Grade). For an ideal $I$ in $A$, we put

$S_{I}:=\left\{n\right.$; There are $f_{1}, \cdots, f_{n} \in I$ which forms an $A$-regular sequence. $\}$, and

$$
\operatorname{grade} I:= \begin{cases}0 & \text { if } S_{I}=\emptyset \\ \max S_{I} & \text { if } S_{I} \text { is a non-empty finite set } \\ +\infty & \text { if } S_{I} \text { is an infinite set. }\end{cases}
$$

Theorem 4.18 (Buchsbaum-Eisenbud [4]). For a complex of free A-modules of finite rank.

$$
F_{\bullet}: 0 \rightarrow F_{s} \stackrel{\phi_{s}}{\rightarrow} F_{s-1} \stackrel{\phi_{s-1}}{\rightarrow} \rightarrow \cdots \rightarrow F_{1} \stackrel{\phi_{5}}{\rightarrow} F_{0} \rightarrow 0,
$$

set $r_{i}:=\sum_{j=i}^{s}(-1)^{j-i} \operatorname{rank} F_{j}$. Then the following are equivalent: 
(1) $F_{\bullet}$ is 0-spherical.

(2) grade $I_{r_{i}}\left(\phi_{i}\right) \geqslant i$ for any $i$ in $(s]$.

Proof of Theorem 4.15. Without loss of generality, we may assume that $S=(n]$ and $x$ is a free non-degenerate Koszul cube associated with an $A$-sequence $f_{1}, \cdots, f_{n}$. We put $m=\operatorname{rank} x$ and

$$
r_{i}=\sum_{j=i}^{n}(-1)^{j-i} \operatorname{rank} \operatorname{Tot} \operatorname{Typ}\left(\mathfrak{f}_{S}\right)_{j}=\sum_{j=i}^{n}(-1)^{j-i}\left(\begin{array}{c}
n \\
j
\end{array}\right) .
$$

Then we have

$$
\sum_{j=i}^{n}(-1)^{j-i} \operatorname{rank} \operatorname{Tot} x=m r_{i}
$$

It is well-known that in this case, the complex $\operatorname{Tot} \operatorname{Typ}\left(\mathfrak{f}_{S}\right)$ is 0 -spherical. (See $[\mathbf{3 0}$, Corollary 4.5.4]). Therefore by 4.18 , it follows that grade $I_{r_{i}}\left(d_{i}^{\operatorname{Tot} \operatorname{Typ}\left(f_{s}\right)}\right) \geqslant i$ for any $i$ in $(n]$. Now inspection shows that for each $i \in(n], I_{r_{i}}\left(d_{i}^{\operatorname{Tot} \operatorname{Typ}\left(\mathfrak{f}_{S}\right)}\right) \subset I_{m r_{i}}\left(d_{i}^{\operatorname{Tot} x}\right)$. Therefore we use Theorem 4.18 again, it turns out that $x$ is 0 -spherical.

Corollary 4.19. A Koszul cube is admissible.

Proof. Let $x$ be a Koszul cube associated with an $A$-sequence $\left\{f_{s}\right\}_{s \in S}$. Since the notion of admissibility is a property of certain exactness of morphisms of modules, we learn that it is a local property. We take a prime ideal $\mathfrak{p}$ of $A$ and put $T:=$ $\left\{s \in S ; f_{s} \in \mathfrak{p}\right\}$. Then by $3.17,4.10,4.12$ and replacing $A, x$ with $A_{\mathfrak{p}},\left(\left.\left(x_{\mathfrak{p}}\right)\right|_{T}\right)_{\text {nondeg }}$, respectively, we shall assume that $A$ is local and $x$ is a non-degenerate free Koszul cube. We are going to prove the assertion by induction of $\# S$ and check that $x$ satisfies the conditions in 3.15 (2). For $\# S=1$, the assertion is trivial. Now we assume that $\# S>1$. Since every faces of $x$ are again non-degenerate free Koszul cubes, by inductive hypothesis, they are admissible. On the other hand, Tot $x$ is 0 -spherical by 4.15 . Therefore we get the result.

Recall the definition for $\ltimes$ from 3.18 and $\mathcal{M}_{A}^{\mathfrak{f}_{T}}(\# T)$ from 4.6.

Corollary 4.20. We have the equality

$$
\operatorname{Kos}_{A}^{\mathfrak{f}_{S}}=\underset{T \in \mathcal{P}(S)}{\ltimes} \mathcal{M}_{A}^{\mathfrak{f}_{T}}(\# T) .
$$

Proof. For any Koszul cube $x$ associated with the $A$-sequence $\left\{f_{s}\right\}_{s \in S}$, we need to check the following two assertions:

(1) $x$ is admissible.

(2) For any $T \in \mathcal{P}(S)$ and $U \in \mathcal{P}(S \backslash T), \mathrm{H}_{0}^{T}(x)_{U}$ is in $\mathcal{M}_{A}^{\mathfrak{f}_{T}}(\# T)$.

Assertion (1) has been proven in 4.19. We prove assertion (2). First let us notice that we have

$$
\operatorname{Supp} \mathrm{H}_{0}^{T}(x)_{U} \subset \bigcap_{t \in T} \operatorname{Supp}_{0}^{t}(x)_{U} \subset \underset{t \in T}{\cap} V\left(f_{t}\right) .
$$

By 3.14, $\operatorname{Tot}\left(\left.x\right|_{T} ^{U}\right)$ is a projective resolution of $\mathrm{H}_{0}^{T}(x)_{U}$ and therefore

$$
\operatorname{Projdim}_{A} \mathrm{H}_{0}^{T}(x)_{U} \leqslant \# T \text {. }
$$

This means that $\mathrm{H}_{0}^{T}(x)_{U}$ is in $\mathcal{M}_{A}^{\mathfrak{f}_{T}}(\# T)$. 


\section{Koszul resolution theorem}

In this section, we will prove the first main theorem 5.14 by utilizing theorem 2.21 . To check the hypothesis in 2.21, the key ingredient is Koszul resolution theorem 5.12 which gives a resolution process of pure weight modules by finite direct sums of typical Koszul cubes. Let us recall that $A$ is a commutative noetherian ring with unit and in this section, let us fix a finite set $S$ and an $A$-sequence $\mathfrak{f}_{S}=\left\{f_{s}\right\}_{s \in S}$ and the letter $p$ means a non-negative integer or $\infty$. Recall the definition of $\mathcal{M}_{A}^{I}(q)$ from 4.6 .

Definition 5.1 (Reduced modules). An $A$-module $M$ in $\mathcal{M}_{A}^{\mathfrak{f}_{S}}$ is said to be reduced if $\mathfrak{f}_{S} M=0$. The full subcategory of reduced $A$-modules is just $\mathcal{M}_{A / \mathfrak{f}_{S} A}$. We write $\mathcal{M}_{A, \text { red }}^{\mathfrak{f}_{S}}(p)$ for the full subcategory of reduced modules in $\mathcal{M}_{A}^{\mathfrak{f}_{S}}(p)$. Since $\mathcal{M}_{A, \text {,red }}^{\mathfrak{f}_{S}}(p)$ is closed under taking sub- and quotient objects in $\mathcal{M}_{A}^{\mathfrak{f}_{S}}(p)$, applying Lemma 5.3 below, we learn that $\mathcal{M}_{A, \text { red }}^{\mathfrak{f}_{S}}(p)$ naturally becomes an exact category. We also write $\mathcal{M}_{A, \text {,red }}^{\mathfrak{f}_{S}}$ for $\mathcal{M}_{A, \text { red }}^{\mathfrak{f}_{S}}(\infty)$.

Notation 5.2. To emphasize the contrast with the index red, we sometimes denote $\mathcal{M}_{A}^{\mathfrak{f}_{S}}(p), \mathbf{K o s}_{A}^{\mathfrak{f}_{S}}$ and so on by $\mathcal{M}_{A, \emptyset}^{\mathfrak{f}_{S}}(p), \mathbf{K o s}_{A, \emptyset}^{\mathfrak{f}_{S}}$, respectively.

Lemma 5.3. Let $\mathcal{E}$ be an exact category and $\mathcal{F}$ a full subcategory which satisfies the following two conditions:

(1) $\mathcal{F}$ is closed under taking finite direct sums. In particular $\mathcal{F}$ has a zero object 0.

(2) $\mathcal{F}$ is closed under taking admissible sub-and quotient objects in $\mathcal{E}$. That is, for an admissible exact sequence in $\mathcal{E}$

$$
x \longmapsto y \rightarrow z,
$$

if $y$ is isomorphic to an object in $\mathcal{F}$, then $x$ and $z$ are also.

Then we can consider $\mathcal{F}$ as an exact category by declaring that a sequence $x \longmapsto y \rightarrow z$ is an admissible exact sequence in $\mathcal{F}$ if and only if it is in $\mathcal{E}$.

Proof. For simplicity, we may suppose that $\mathcal{F}$ is closed under isomorphisms in $\mathcal{E}$. Namely for an object $x$ in $\mathcal{F}$ and an object $y$ in $\mathcal{E}$, if $x \stackrel{\sim}{\rightarrow} y$, then $y$ is also in $\mathcal{F}$. We shall just check that the class of admissible monomorphisms (resp. admissible epimorphisms) in $\mathcal{F}$ is closed under compositions and co-base (resp. base) change by arbitrary morphisms. We only check for the admissible monomorphisms case. For the admissible epimorphisms case, almost the same arguments work.

Let $x \longmapsto y$ and $y \longmapsto z$ be admissible monomorphisms in $\mathcal{F}$. By $(2), z / x$ can be taken in $\mathcal{F}$. Therefore the sequence

$$
x \longmapsto z \rightarrow z / x
$$

is an admissible exact sequence in $\mathcal{E}$ and the composition $x \longmapsto z$ is an admissible monomorphism in $\mathcal{F}$. 
Next consider the following commutative diagram in $\mathcal{E}$ :

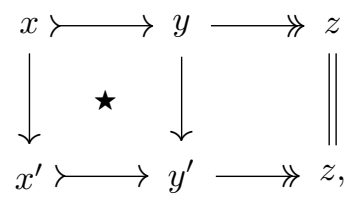

where the square $\star$ is coCartesian and we assume that $x, y, z$ and $x^{\prime}$ are in $\mathcal{F}$. Then by $[\mathbf{1 6}$, p.406 step 1], we have an admissible exact sequence

$$
x \longmapsto x^{\prime} \oplus y \rightarrow y^{\prime} .
$$

By (1), $x^{\prime} \oplus y$ is in $\mathcal{F}$ and by (2), $y^{\prime}$ is also in $\mathcal{F}$.

Recall the definition of $\ltimes$ from 3.18 .

Definition 5.4. Let $S=U \sqcup V$ be a disjoint decomposition of $S$.

(1) We define the categories $\mathcal{M}_{A}\left(\mathfrak{f}_{U} ; \mathfrak{f}_{V}\right)(p), \mathcal{M}_{A, \text { red }}\left(\mathfrak{f}_{U} ; \mathfrak{f}_{V}\right)(p)$ which are full subcategories of $\mathbf{C u b}^{V} \mathcal{M}_{A}$ by

$$
\mathcal{M}_{A, ?}\left(\mathfrak{f}_{U} ; \mathfrak{f}_{V}\right)(p)=\underset{T \in \mathcal{P}(V)}{\ltimes} \mathcal{M}_{A, ?}^{\mathfrak{f}_{U \sqcup T}}(p+\# T),
$$

where $?=\emptyset$ or red. In particular, we write $\operatorname{Kos}_{A, \text { red }}^{\mathfrak{f}_{S}}$ for $\mathcal{M}_{A, \text { red }}\left(\mathfrak{f}_{\emptyset} ; \mathfrak{f}_{S}\right)(0)$. This notation is compatible with the equality (7) in 5.5 (3). A cube in $\operatorname{Kos}_{A, \text { red }}^{\mathfrak{f}_{S}}$ is said to be a reduced Koszul cube (associated with an $A$-sequence $\left\{f_{s}\right\}_{s \in S}$ ).

(2) A morphism $f: x \rightarrow y$ in $\mathcal{M}_{A, ?}\left(\mathfrak{f}_{U} ; \mathfrak{f}_{V}\right)(p)$ is a (total) quasi-isomorphism if Tot $f$ is a quasi-isomorphism. We write $\operatorname{tq}\left(\mathcal{M}_{A, ?}\left(\mathfrak{f}_{U} ; \mathfrak{f}_{V}\right)(p)\right)$ or simply tq for the class of total quasi-isomorphisms in $\mathcal{M}_{A, ?}\left(\mathfrak{f}_{U} ; \mathfrak{f}_{V}\right)(p)$.

Remark 5.5. In the notation above, using 3.20, we have the following:

(1) $\mathcal{M}_{A}\left(\mathfrak{f}_{U} ; \mathfrak{f}_{V}\right)(p)$ is closed under extensions in $\mathbf{C u b}^{V} \mathcal{M}_{A}$. In particular it becomes an exact category in the natural way.

(2) $\mathcal{M}_{A \text {,red }}\left(\mathfrak{f}_{U} ; \mathfrak{f}_{V}\right)(p) \hookrightarrow \mathcal{M}_{A}\left(\mathfrak{f}_{U} ; \mathfrak{f}_{V}\right)(p)$ is closed under taking finite direct sums and admissible sub- and quotient objects. In particular, $\mathcal{M}_{A, \text { red }}\left(\mathfrak{f}_{U} ; \mathfrak{f}_{V}\right)(p)$ naturally becomes an exact category by virtue of 5.3 .

(3) By 4.20, we have the equality

$$
\mathcal{M}_{A}\left(\mathfrak{f}_{\emptyset} ; \mathfrak{f}_{S}\right)(0)=\operatorname{Kos}_{A}^{\mathfrak{f}_{S}} .
$$

(4) By definitions of multi semi-direct products in $3.18(1)$ and $\mathcal{M}_{A, ?}\left(\mathfrak{f}_{U} ; \mathfrak{f}_{V}\right)(p)$, cubes in $\mathcal{M}_{A, ?}\left(\mathfrak{f}_{U} ; \mathfrak{f}_{V}\right)(p)$ are admissible. A morphism $f: x \rightarrow y$ in $\mathcal{M}_{A, ?}\left(\mathfrak{f}_{U} ; \mathfrak{f}_{V}\right)(p)$ is a total quasi-isomorphism if and only if $\mathrm{H}_{0}^{V}(f)$ is an isomorphism by $3.13(2)$.

(5) We put $\mathcal{F}_{T}:=\mathcal{M}_{A}^{\mathfrak{f}_{T}}(p+\# T)$ for any $T \in \mathcal{P}(S)$ and $\mathfrak{F}=\left\{\mathcal{F}_{T}\right\}_{T \in \mathcal{P}(S)}$ and for any disjoint decomposition $S=U \sqcup V$, we put $\left.\mathfrak{F}\right|_{V} ^{U}:=\left\{\mathcal{F}_{U \sqcup T}\right\}_{T \in \mathcal{P}(V)}$. Then since we have the equality $\left.\ltimes \mathfrak{F}\right|_{V} ^{U}=\mathcal{M}_{A, ?}\left(\mathfrak{f}_{U} ; \mathfrak{f}_{V}\right)(p+\# U)$, the family $\mathfrak{F}$ satisfies the condition 3.21. 
Remark 5.6. Let $S=U \sqcup V$ be a disjoint decomposition of $S$ with $V \neq \emptyset$. Then for any $v \in V$, by 3.19 (1) and (3), we have the following equalities

$$
\begin{gathered}
\mathcal{M}_{A, ?}\left(\mathfrak{f}_{U} ; \mathfrak{f}_{V}\right)(p)=\mathcal{M}_{A, ?}\left(\mathfrak{f}_{U \sqcup\{v\}} ; \mathfrak{f}_{V \backslash\{v\}}\right)(p+1) \ltimes \mathcal{M}_{A, ?}\left(\mathfrak{f}_{U} ; \mathfrak{f}_{V \backslash\{v\}}\right)(p) \\
\mathcal{M}_{A, ?}\left(\mathfrak{f}_{U} ; \mathfrak{f}_{V}\right)(p)^{\mathrm{tq}}=\mathcal{M}_{A, ?}\left(\mathfrak{f}_{U \sqcup\{v\}} ; \mathfrak{f}_{V \backslash\{v\}}\right)(p+1)^{\mathrm{tq}} \ltimes \mathcal{M}_{A, ?}\left(\mathfrak{f}_{U} ; \mathfrak{f}_{V \backslash\{v\}}\right)(p)
\end{gathered}
$$

for $?=\emptyset$ or red.

To prove Proposition 5.8, we need to recall the following facts:

Review 5.7. Notice that for a short exact sequence of $A$-modules

$$
0 \rightarrow N \rightarrow N^{\prime} \rightarrow N^{\prime \prime} \rightarrow 0,
$$

we can easily prove the following assertions by utilizing the Ext-criterion of projective dimensions. (see [30, pd Lemma 4.1.6]).

(1) If $\operatorname{Projdim}_{A} N^{\prime \prime} \leqslant n+1$ and $\operatorname{Projdim}_{A} N^{\prime} \leqslant n$, then $\operatorname{Projdim}_{A} N \leqslant n$.

(2) If $\operatorname{Projdim}_{A} N \leqslant n$ and $\operatorname{Projdim}_{A} N^{\prime \prime} \leqslant n$, then $\operatorname{Projdim}_{A} N^{\prime} \leqslant n$.

Proposition 5.8. Let $n$ be an integer such that $\# S \leqslant n<\infty$ and $?=\emptyset$ or red. Then (1) $\mathcal{M}_{A, ?}^{\mathfrak{f}_{S}}(n)$ is closed under extensions and taking kernels of admissible epimorphisms in $\mathcal{M}_{A, ?}^{\mathfrak{f}_{S}}(n+1)$.

(2) Moreover the inclusion functor of opposite categories

$$
\left(\mathcal{M}_{A, ?}^{\mathfrak{f}_{S}(n)}\right)^{o p} \hookrightarrow\left(\mathcal{M}_{A, ?}^{\mathfrak{f}_{S}}(n+1)\right)^{o p}
$$

satisfies the resolution conditions in 1.9. In particular, we have a homotopy equivalence on K-theory:

$$
K\left(\mathcal{M}_{A, ?}^{\mathfrak{f}_{S}}(n)\right) \rightarrow K\left(\mathcal{M}_{A, ?}^{\mathfrak{f}_{S}}(n+1)\right) .
$$

Proof. For (1), we need only check the projective dimension conditions and they are easily done by (1) and (2) in 5.7. For (2), we need to prove the following two assertions for $?=\emptyset$ and red, respectively.

(a) For any $M \in \mathcal{M}_{A}^{\mathfrak{f}_{S}}(n+1)$, there exists an $A$-module $N$ in $\mathcal{M}_{A, ?}^{\mathfrak{f}_{S}}(n)$ and an admissible epimorphism $N \rightarrow M$.

(b) For any admissible short exact sequence $L \nrightarrow N \rightarrow M$ in $\mathcal{M}_{A, ?}^{\mathfrak{f}_{S}}(n+1)$, if $N$ and $M$ are in $\mathcal{M}_{A, ?}^{\mathfrak{f}_{S}}(n)$, then $L$ is also in $\mathcal{M}_{A, ?}^{\mathfrak{f}_{S}}(n)$.

Proof of assertion (a): For any $M \in \mathcal{M}_{A}^{\mathfrak{f}_{S}}(n)$, there are positive integers $t_{s}$ such that $f_{s}^{t_{s}} M=0$ for any $s \in S$. We put $g_{s}:=f_{s}^{t_{s}}$ and $B:=A / J$, where $J$ is the ideal generated by $\left\{g_{s}\right\}_{s \in S}$. Therefore we can consider $M$ to be a finitely generated $B$-module and there is a surjection from a finitely generated free $B$-module $N$ to $M$. Since $\operatorname{Projdim}_{A} N=\# S, N$ is in $\mathcal{M}_{A}^{\mathfrak{f}_{S}}(n)$. If $M$ is reduced, we can take $t_{s}=1$ for each $s \in S$. Then in this case $N$ is also reduced.

Proof of assertion (b): We get the result by 5.7 (1) for $?=\emptyset$. If $N$ is reduced, then $L$ is also reduced.

Corollary 5.9. For $?=\emptyset$ or red, any disjoint decomposition $S=U \sqcup V$ and any integer $p \geqslant \# U, \mathcal{M}_{A, ?}\left(\mathfrak{f}_{U} ; \mathfrak{f}_{V}\right)(p)$ is closed under extensions and taking kernels of admissible epimorphisms in $\mathcal{M}_{A, ?}\left(\mathfrak{f}_{U} ; \mathfrak{f}_{V}\right)(p+1)$. 
Proof. By virtue of 3.20 (2), we need only check that $\mathcal{M}_{A, ?}^{\mathfrak{f}_{T} \sqcup U}(p+\# T)$ is closed under extensions and taking kernels of admissible epimorphisms in $\mathcal{M}_{A, ?}^{\mathfrak{f}_{T} \sqcup \mathrm{T}}(p+1+\# T)$ for any $T \in \mathcal{P}(V)$. This was done in 5.8 .

Remark 5.10. Let us assume that $A$ is Cohen-Macaulay. Recall that a commutative noetherian ring $C$ is Cohen-Macaulay if and only if every ideal of height at least $p$ contains an $C$-regular sequence of length $p$. (See $[\mathbf{3}, \S 2.5$, Proposition. 7].) Notice that the ordered set $X$ of all ideals of $A$ that contains an $A$-regular sequence of length $p$ with usual inclusion is directed. Therefore $\mathcal{M}_{A}^{p}(p)$ is the filtered limit $\lim _{\mathfrak{g} S} \mathcal{M}_{A}^{\mathfrak{g}_{S}}(p)$, where $\mathfrak{g}_{S}$ runs through any regular sequence such that $\# S=p$.

Corollary 5.11. Let us assume that $A$ is regular. Then for any natural number $p$, the inclusion functor $\mathcal{M}_{A}^{p}(p) \hookrightarrow \mathcal{M}_{A}^{p}$ induces a homotopy equivalence on $K$-theory:

$$
K\left(\mathcal{M}_{A}^{p}(p)\right) \rightarrow K\left(\mathcal{M}_{A}^{p}\right) .
$$

Proof. By regularity of $A$, we may ignore the projective dimension condition in 5.8 (2). The assertion follows from 5.8 (2) and 5.10.

Theorem 5.12 (Koszul resolution theorem). Let $n$ be a non-negative integer, $S=U \sqcup V$ a disjoint decomposition of $S$ and $p \geqslant \# U$ an integer. Fix an object $z \in \mathcal{H O M}\left([n], \mathcal{M}_{A}\left(\mathfrak{f}_{U} ; \mathfrak{f}_{V}\right)(p+1)\right)$,

$$
z: z(0) \rightarrow z(1) \rightarrow \cdots \rightarrow z(n) .
$$

(1) For each $s \in S$, there is a family of non-negative integers $\left\{m_{s}\right\}_{s \in S}$ such that $f_{u}^{m_{u}} z(j)_{T}=0$ for any $T \in \mathcal{P}(V), j \in[n]$ and $u \in U$ and $f_{v}^{m_{v}} \mathrm{H}_{0}(\operatorname{Tot}(z(j)))=0$ for any $j \in[n]$ and $v \in V$. We put $g_{s}=f_{s}^{m_{s}}$ for each $s \in S$ and $B:=A / \mathfrak{g}_{U}$, where $\mathfrak{g}_{U}$ is the ideal generated by $\left\{g_{u}\right\}_{u \in U}$.

(2) There is an object $y \in \mathcal{H O M}\left([n], \mathcal{M}_{A}\left(\mathfrak{f}_{U} ; \mathfrak{f}_{V}\right)(\# U)\right)$ such that for each $i \in[n]$, $y(i)$ is of the following form:

$$
y(i)=\bigoplus_{T \in \mathcal{P}(V)} \operatorname{Typ}_{B}\left(\mathfrak{g}_{V}^{T}\right)^{\oplus l_{T}(i)}
$$

and such that there is an admissible epimorphism $y \rightarrow z$. Here the notation $\mathfrak{g}_{V}^{T}$ means the family $\left\{g_{v}^{\chi_{T}(v)}\right\}_{v \in V}$, where $\chi_{T}$ is the characteristic function associated with $T$ (see 2.2) and for the definition of the typical Koszul cube Typ, see 2.7.

Proof. Since $z(j)_{T}$ is in $\mathcal{M}_{A}^{\mathfrak{f}_{U}}$ for any $T \in \mathcal{P}(V)$ and $\mathrm{H}_{0}(\operatorname{Tot}(z(j)))$ is in $\mathcal{M}_{A}^{\mathfrak{f}_{S}}$, assertion (1) is trivial. We are concentrating on proving assertion (2). We first prove that for the case $n=0$ by induction on $\# V$. In this case, we consider $z$ to be an object in $\mathcal{M}_{A}\left(\mathfrak{f}_{U} ; \mathfrak{f}_{V}\right)(p+1)$. Let us assume that $V=\emptyset$. Since $z$ is a finitely generated $B$ module, there is an integer $l_{1}$ and a surjection $B^{\oplus l_{1}} \rightarrow z$. We put $y=B^{\oplus l_{1}}$ and by 5.8 , the map $y \rightarrow z$ is an admissible epimorphism. Next we assume that $\# V \geqslant 1$ and let us fix an element $v \in V$ and an object $z \in \mathcal{M}_{A}\left(\mathfrak{f}_{U} ; \mathfrak{f}_{V}\right)(p+1)$. Then by the formula 5.6, we can consider $z$ to be a complex $\left[z_{1} \stackrel{d^{z}}{\rightarrow} z_{0}\right]$ in $\mathcal{M}_{A}\left(\mathfrak{f}_{U} ; \mathfrak{f}_{V \backslash\{v\}}\right)(p+1)$. By the inductive hypothesis, there is an admissible epimorphism $y^{\prime} \rightarrow z_{0}$, where $y^{\prime}$ is 
of the form

$$
y^{\prime}=\bigoplus_{T \in \mathcal{P}(V \backslash\{v\})} \operatorname{Typ}_{B}\left(\mathfrak{g}_{V \backslash\{v\}}^{T}\right)^{\oplus l_{1, T}} .
$$

Therefore by the cube lemma 2.8, we get a term-wised surjection morphism $y^{\prime \prime} \rightarrow \mathrm{H}_{0} z$, where $y^{\prime \prime}$ is of the form

$$
y^{\prime \prime}=\bigoplus_{T \in \mathcal{P}(V \backslash\{v\})} \operatorname{Typ}_{B /\left(g_{v}\right)}\left(\mathfrak{g}_{V \backslash\{v\}}^{T}\right)^{\oplus l_{1, T}}
$$

and it makes the diagram below commutative:

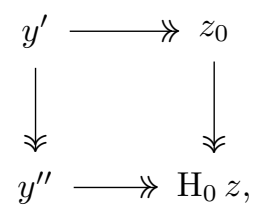

where the vertical maps are the canonical projections. Therefore we get a map $y^{\prime} \rightarrow z_{1}$ which makes the diagram below commutative:

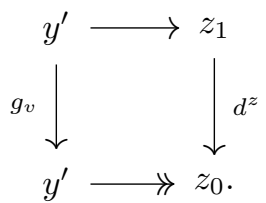

By the induction hypothesis, there is a term-wised surjective morphism $y^{\prime} \oplus y^{\prime \prime \prime} \rightarrow z_{1}$, where $y^{\prime \prime \prime}$ is of the form

$$
y^{\prime \prime \prime}=\bigoplus_{W \in \mathcal{P}(V \backslash\{v\})} \operatorname{Typ}_{B}\left(\mathfrak{g}_{V \backslash\{v\}}^{W}\right)^{\oplus l_{0, W}}
$$

and it makes the diagram below commutative:

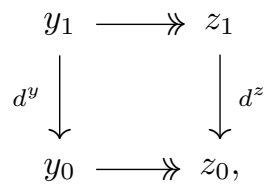

where $y_{i}=y^{\prime} \oplus y^{\prime \prime \prime}$ for $i=0,1$ and $d^{y}=\left(\begin{array}{cc}g_{v} & 0 \\ 0 & 1\end{array}\right)$ and we put $y=\left[y_{1} \stackrel{d^{y}}{\rightarrow} y_{0}\right]$. Thus by 5.9 , we learn that $y \rightarrow z$ is an admissible epimorphism and therefore we get the conclusion for the case of $n=0$. Next we consider the case of general $n$. For each $z$ and each $i \in[n]$, by the previous argument, we have $y(i)=\underset{T \in \mathcal{P}(V)}{\bigoplus} \operatorname{Typ}_{B}\left(\mathfrak{g}_{V}^{T}\right)^{\oplus l_{T}(i)}$ for a suitable non-negative integer $l_{T}(i)$ and an admissible epimorphism $y(i) \rightarrow z(i)$. So we need only prove that for each $i \in[n-1]$, there is a map $y(i) \rightarrow y(i+1)$ which 
makes diagram below commutative:

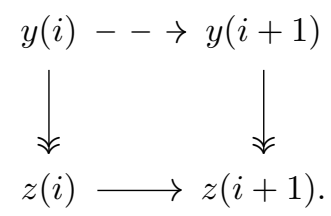

Since $y(i)$ is in $\underset{T \in \mathcal{P}(V)}{\ltimes} \mathcal{P}_{B / \mathfrak{g}_{T}}$, by applying $3.20(3)$ to $\mathcal{F}_{T}=\mathcal{P}_{B / \mathfrak{g}_{T}}$ and $\mathcal{E}_{T}=\mathcal{M}_{B \text {,red }}^{\mathfrak{g}_{T}}$ for each $T \in \mathcal{P}(V)$, we learn that $y(i)$ is a projective object in $\underset{T \in \mathcal{P}(V)}{\ltimes} \mathcal{M}_{B \text {,red }}^{\mathfrak{g}_{T}}$. Moreover since $y(i+1), z(i+1)$ are in $\underset{T \in \mathcal{P}(V)}{\ltimes} \mathcal{M}_{B \text {,red }}^{\mathfrak{g}_{T}}$, there is the dotted map in the commutative diagram above by projectivity of $y(i)$.

Recall the definition of strongly adroit systems from 2.20 and the definition of tq from 5.4.

Corollary 5.13. For $?=\emptyset$ or red, any decomposition $S=U \sqcup V$ with $V \neq \emptyset$, any element $v$ in $V$ and any integer $p \geqslant \# U$, triples

$$
\mathcal{X}=\left(\mathcal{M}_{A, ?}\left(\mathfrak{f}_{U} ; \mathfrak{f}_{V \backslash\{v\}}\right)(p), \mathcal{M}_{A, ?}\left(\mathfrak{f}_{U} ; \mathfrak{f}_{V \backslash\{v\}}\right)(p+1), \mathcal{M}_{A, ?}\left(\mathfrak{f}_{U \sqcup\{v\}} ; \mathfrak{f}_{V \backslash\{v\}}\right)(p+1)\right)
$$

and

$\mathcal{X}^{\prime}=\left(\mathcal{M}_{A, ?}\left(\mathfrak{f}_{U} ; \mathfrak{f}_{V \backslash\{v\}}\right)(p), \mathcal{M}_{A, ?}\left(\mathfrak{f}_{U} ; \mathfrak{f}_{V \backslash\{v\}}\right)(p+1), \mathcal{M}_{A, ?}\left(\mathfrak{f}_{U \sqcup\{v\}} ; \mathfrak{f}_{V \backslash\{v\}}\right)(p+1)^{\mathrm{tq}}\right)$ are strongly adroit systems.

Proof. Consider the triple

$$
\begin{aligned}
\mathcal{E}_{1} & :=\mathcal{M}_{A, ?}\left(\mathfrak{f}_{U} ; \mathfrak{f}_{V \backslash\{v\}}\right)(p), \\
\mathcal{E}_{2} & :=\mathcal{M}_{A, ?}\left(\mathfrak{f}_{U} ; \mathfrak{f}_{V \backslash\{v\}}\right)(p+1) \text { and } \\
\mathcal{F} & :=\mathcal{M}_{A, ?}\left(\mathfrak{f}_{U \sqcup\{v\}} ; \mathfrak{f}_{V \backslash\{v\}}\right)(p+1) .
\end{aligned}
$$

Claim. $\mathcal{F}$ is contained in $\mathcal{E}_{2}$.

Proof of claim. If $V=\{v\}$, then $\mathcal{E}_{2}=\mathcal{M}_{A, ?}^{\mathfrak{f} U}(p+1), \mathcal{F}=\mathcal{M}_{A, \text { ? }}^{\mathfrak{f} U \sqcup\}}(p+1)$ and therefore we get the assertion. If $\# V \geqslant 2$, then let us fix an element $v^{\prime} \in V \backslash\{v\}$. Then by 5.6 , we have equalities

$$
\begin{gathered}
\mathcal{E}_{2}=\mathcal{M}_{A, ?}\left(\left(\mathfrak{f}_{U \sqcup\left\{v^{\prime}\right\}} ; \mathfrak{f}_{V \backslash\left\{v, v^{\prime}\right\}}\right)(p+2) \ltimes \mathcal{M}_{A, ?}\left(\mathfrak{f}_{U \sqcup\left\{v^{\prime}\right\}} ; \mathfrak{f}_{V \backslash\left\{v, v^{\prime}\right\}}\right)(p+1)\right. \text { and } \\
\mathcal{F}=\mathcal{M}_{A, ?}\left(\mathfrak{f}_{U \sqcup\left\{v, v^{\prime}\right\}} ; \mathfrak{f}_{V \backslash\left\{v, v^{\prime}\right\}}\right)(p+2) \ltimes \mathcal{M}_{A, ?}\left(\mathfrak{f}_{U \sqcup\left\{v, v^{\prime}\right\}} ; \mathfrak{f}_{V \backslash\left\{v, v^{\prime}\right\}}\right)(p+2) .
\end{gathered}
$$

Hence we learn that $\mathcal{F}$ is contained in $\mathcal{E}_{2}$.

Since $\mathcal{F}$ is closed under extensions (resp. sub- and quotient objects) in $\mathbf{C u b}^{V \backslash\{v\}} \mathcal{M}_{A}$ if $?=\emptyset($ resp. ? = red $), \mathcal{F} \ltimes \mathcal{E}_{i}(i=1,2)$ are strict exact subcategories of $\mathbf{C u b}^{V} \mathcal{M}_{A}$ by 2.10. The conditions (Adr 2) and (Adr 3) follow from 5.9. Finally the condition (Adr 5) follows from 5.12. The proof for $\mathcal{X}^{\prime}$ is similar. Therefore we get the result.

Corollary 5.14. For $?=\emptyset$ or red, any decomposition $S=U \sqcup V$ and any integer $p \geqslant \# U$, we have the following: 
(1) (Local weight theorem). Assume that $V$ is a non-empty set and $v$ is an element in $V$. Then the exact functor

$$
\mathrm{H}_{0}^{v}:\left(\mathcal{M}_{A, ?}\left(\mathfrak{f}_{U} ; \mathfrak{f}_{V}\right)(p), \mathrm{tq}\right) \rightarrow\left(\mathcal{M}_{A, ?}\left(\mathfrak{f}_{U \sqcup\{v\}} ; \mathfrak{f}_{V \backslash\{v\}}\right)(p+1), \mathrm{tq}\right)
$$

induces a homotopy equivalence on $K$-theory:

$$
K\left(\mathrm{H}_{0}^{v}\right): K\left(\mathcal{M}_{A, ?}\left(\mathfrak{f}_{U} ; \mathfrak{f}_{V}\right)(p) ; \mathrm{tq}\right) \rightarrow K\left(\mathcal{M}_{A, ?}\left(\mathfrak{f}_{U \sqcup\{v\}} ; \mathfrak{f}_{V \backslash\{v\}}\right)(p+1) ; \mathrm{tq}\right) .
$$

In particular the exact functor

$$
\mathrm{H}_{0} \text { Tot: }\left(\mathcal{M}_{A, ?}\left(\mathfrak{f}_{U} ; \mathfrak{f}_{V}\right)(p), \mathrm{tq}\right) \rightarrow \mathcal{M}_{A, ?}^{\mathfrak{f}_{S}}(p+\# V)
$$

induces a homotopy equivalence on $K$-theory:

$$
K\left(\mathcal{M}_{A, ?}\left(\mathfrak{f}_{U} ; \mathfrak{f}_{V}\right)(p), \mathrm{tq}\right) \rightarrow K\left(\mathcal{M}_{A, ?}^{\mathfrak{f}_{S}}(p+\# V)\right) .
$$

In particular the exact functor $\mathrm{H}_{0}$ Tot: $\left(\operatorname{Kos}_{A, ?}^{\mathfrak{f}_{S}}, \mathrm{tq}\right) \rightarrow \mathcal{M}_{A, ?}^{\mathfrak{f}_{S}}(\# S)$ induces a homotopy equivalence on $K$-theory:

$$
K\left(\operatorname{Kos}_{A, ?}^{\mathfrak{f}_{S}}, \mathrm{tq}\right) \rightarrow K\left(\mathcal{M}_{A, ?}^{\mathfrak{f}_{S}}(\# S)\right) .
$$

(2) The exact functors

$$
\begin{aligned}
\lambda: \mathcal{M}_{A, ?}\left(\mathfrak{f}_{U} ; \mathfrak{f}_{V}\right)(p) & \rightarrow \prod_{T \in \mathcal{P}(V)} \mathcal{M}_{A, ?}^{\mathfrak{f} U \sqcup T}(p+\# T) \text { and } \\
\lambda^{\prime}: \mathcal{M}_{A, ?}\left(\mathfrak{f}_{U} ; \mathfrak{f}_{V}\right)(p)^{t q} & \rightarrow \prod_{T \in \mathcal{P}(V) \backslash\{V\}} \mathcal{M}_{A, ?}^{\mathfrak{f}_{U} \sqcup T}(p+\# T)
\end{aligned}
$$

which sends an object $x$ to $\left(\mathrm{H}_{0}^{U \sqcup T}(x)\right)_{T \in \mathcal{P}(V)}$ and $\left(\mathrm{H}_{0}^{U \sqcup T}(x)\right)_{T \in \mathcal{P}(V) \backslash\{V\}}$, respectively, induce homotopy equivalences on $K$-theory:

$$
\begin{aligned}
K\left(\mathcal{M}_{A, ?}\left(\mathfrak{f}_{U} ; \mathfrak{f}_{V}\right)(p)\right) & \rightarrow \prod_{T \in \mathcal{P}(V)} K\left(\mathcal{M}_{A, ?}^{\mathfrak{f}_{U \sqcup T}}(p+\# T)\right) \text { and } \\
K\left(\mathcal{M}_{A, ?}\left(\mathfrak{f}_{U} ; \mathfrak{f}_{V}\right)(p)^{\mathrm{tq}}\right) & \rightarrow \prod_{T \in \mathcal{P}(V) \backslash\{V\}} K\left(\mathcal{M}_{A, ?}^{\mathfrak{f}_{U \cup T}}(p+\# T)\right) .
\end{aligned}
$$

(3) (Split fibration theorem). The inclusion functors

$$
\mathcal{M}_{A, ?}\left(\mathfrak{f}_{U} ; \mathfrak{f}_{V}\right)(p)^{\mathrm{tq}} \hookrightarrow \mathcal{M}_{A, ?}\left(\mathfrak{f}_{U} ; \mathfrak{f}_{V}\right)(p)
$$

and the identity functor on $\mathcal{M}_{A, ?}\left(\mathfrak{f}_{U} ; \mathfrak{f}_{V}\right)(p)$ induce a split fibration sequence:

$$
K\left(\mathcal{M}_{A, ?}\left(\mathfrak{f}_{U} ; \mathfrak{f}_{V}\right)(p)^{\mathrm{tq}}\right) \rightarrow K\left(\mathcal{M}_{A, ?}\left(\mathfrak{f}_{U} ; \mathfrak{f}_{V}\right)(p)\right) \rightarrow K\left(\mathcal{M}_{A, ?}\left(\mathfrak{f}_{U} ; \mathfrak{f}_{V}\right)(p) ; \mathrm{tq}\right) .
$$

In particular we have a split fibration sequence:

$$
K\left(\operatorname{Kos}_{A, ?}^{\mathfrak{f}_{S}{ }^{\mathrm{tq}}}\right) \rightarrow K\left(\operatorname{Kos}_{A, ?}^{\mathfrak{f}_{S}}\right) \rightarrow K\left(\operatorname{Kos}_{A, ?}^{\mathfrak{f}_{S}} ; \mathrm{tq}\right) .
$$

Proof. Proof of assertion (1): Consider the strongly adroit system $\mathcal{X}=\left(\mathcal{E}_{1}, \mathcal{E}_{2}, \mathcal{F}\right)$ in (the proof of) 5.13 . We have an equality $\mathcal{F} \ltimes \mathcal{E}_{1}=\mathcal{M}_{A \text {,? }}\left(\mathfrak{f}_{U} ; \mathfrak{f}_{V}\right)(p)$ by 5.6. We apply 2.21 (2) to $\mathcal{X}$ and obtain the proof. The second assertion comes from the isomorphism $\mathrm{H}_{0}$ Tot $\stackrel{\sim}{\rightarrow} \mathrm{H}_{0}^{V}$ by $3.21,5.5$ (5) and the first assertion. The third result follows from the equality (7) in 5.5 (3) and the second assertion. 
Proof of assertion (2): We proceed by induction on the cardinality of $V$. If $V=\emptyset$, then $\mathcal{M}_{A, ?}\left(\mathfrak{f}_{U} ; \mathfrak{f}_{V}\right)(p)=\mathcal{M}_{A, ?}^{\mathfrak{f}_{U}}(p)$ and $\mathcal{M}_{A, ?}\left(\mathfrak{f}_{U} ; \mathfrak{f}_{V}\right)(p)^{\text {tq }}=\{0\}$. Therefore the assertion is trivial. If $\# V \geqslant 1$ and $v$ is an element in $V$, then by the equalities in $5.6 \lambda$ and $\lambda^{\prime}$ have the factorizations

$$
\begin{gathered}
K\left(\mathcal{M}_{A, ?}\left(\mathfrak{f}_{U} ; \mathfrak{f}_{V}\right)(p)\right) \stackrel{\mathbf{I}}{\rightarrow} K\left(\mathcal{M}_{A, ?}\left(\mathfrak{f}_{U \sqcup\{v\}} ; \mathfrak{f}_{V \backslash\{v\}}\right)(p+1)\right) \times K(X) \underset{T \in \mathcal{P}(V)}{\stackrel{\operatorname{II}}{\rightarrow}} K\left(\mathcal{M}_{A, ?}^{\mathfrak{f} U \sqcup T}(p+\# T)\right) \\
\left.K\left(\mathcal{M}_{A, ?}\left(\mathfrak{f}_{U} ; \mathfrak{f}_{V}\right)(p)^{\mathrm{tq}}\right) \stackrel{\mathbf{I}}{\rightarrow} K\left(\mathcal{M}_{A, ?}\left(\mathfrak{f}_{U \sqcup\{v\}} ; \mathfrak{f}_{V \backslash\{v\}}\right)(p+1)^{\mathrm{tq}}\right) \times K(X) \underset{T \in \mathcal{P}(V) \backslash\{V\}}{\rightarrow} \prod_{A, ?} \prod_{\mathcal{M}^{\mathfrak{f}} U \sqcup T}(p+\# T)\right),
\end{gathered}
$$

where $X$ denotes $\mathcal{M}_{A, ?}\left(\mathfrak{f}_{U} ; \mathfrak{f}_{V \backslash\{v\}}\right)(p)$, and the maps $\mathbf{I}$ and $\mathbf{I I}$ are homotopy equivalences by 2.21 (3) and the inductive hypothesis, respectively. Hence we get the result.

Proof of assertion (3): Let us consider the commutative diagram below:

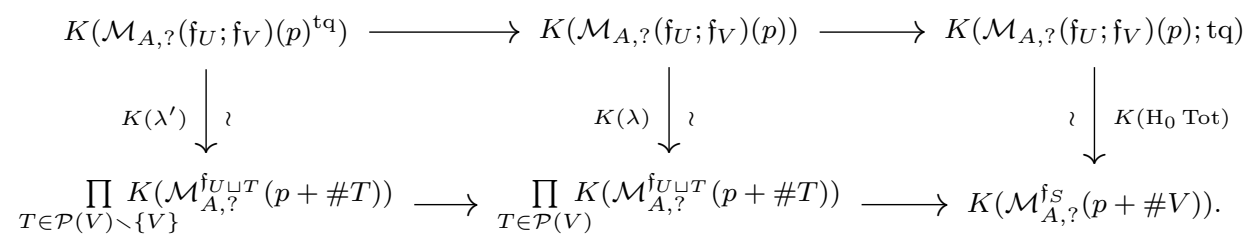

Here vertical maps are homotopy equivalence by (1) and (2) and the bottom horizontal line is a split fibration sequence. Hence we get the result.

Recall the definition of $\mathcal{M}_{A}^{I}(p)$ from 4.6 and $\operatorname{Kos}_{A}^{\mathfrak{f}_{S}}$ from 4.8.

Proof of Theorem 0.2. Since $A$ is local, every $A$-regular sequence is an $A$-sequence by 4.1 (4) (i). Let us assume that $\# S=p$ and let $\mathfrak{f}_{S}=\left\{f_{s}\right\}_{s \in S}$ be an $A$-sequence. Then the exact functor $\mathrm{H}_{0}$ Tot: $\left(\operatorname{Kos}_{A}^{\mathfrak{f}_{S}}, \mathrm{tq}\right) \rightarrow\left(\mathcal{M}_{A}^{\mathfrak{f}_{S}}(p), i\right)$ induces a homotopy equivalence on $K$-theory by 5.14 (1). On the other hand we have the homotopy equivalence $K\left(\mathcal{M}_{A}^{p}(p)\right) \stackrel{\widetilde{\sim}}{\rightarrow} \lim _{\longrightarrow} K\left(\mathcal{M}_{A}^{\mathfrak{g}_{S}}(p)\right)$, where $V\left(\mathfrak{g}_{S}\right) \hookrightarrow$ Spec $A$ runs over the regular closed immersion of codimension $p$ by 5.10. Therefore the Grothendieck group $K_{0}\left(\mathcal{M}_{A}^{p}(p)\right)$ is generated by modules of the form

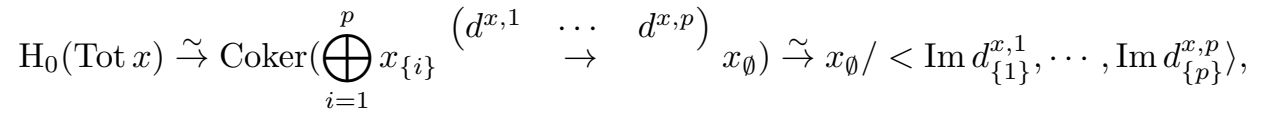

where $x$ is a non-degenerate free Koszul cube associated with some $A$-sequence $g_{1}, \cdots, g_{p}$ and $d_{\{i\}}^{x, i}: x_{\{i\}} \rightarrow x_{\emptyset}$ is a boundary morphism of $x$. Since the sequence $\operatorname{det} d_{\{1\}}^{x, 1}, \cdots, \operatorname{det} d_{\{p\}}^{x, p}$ forms an $A$-sequence by 4.14 , we obtain the result.

\section{A dévissage theorem for $K$-theory of Koszul cubes on regular rings}

In this section, we assume that $A$ is a commutative regular noetherian ring with unit and that the global homological dimension of $A$ is $n$ and $S$ is a finite set. Let us fix an $A$-sequence $\left\{f_{s}\right\}_{s \in S}$ and let $I$ be the ideal in $A$ generated by $\left\{f_{s}\right\}_{s \in S}$. The aim of this section is to prove a dévissage theorem 6.3 for Koszul cubes on $A$. 
Recall from 4.6 and 5.1 that $\mathcal{M}_{A}^{I}(p)$ is the category of finitely generated $A$-modules of projective dimension $\leqslant p$ and Supp $M \subset V(I)$ and $\mathcal{M}_{A \text {.red }}^{I}(p)$ is the full subcategory of modules $M$ with $I M=0$, and that $\# S=\operatorname{Projdim}_{A} A / I$.

Proposition 6.1. For any integer $p \geqslant \# S$, the inclusion functor $\iota: \mathcal{M}_{A, \mathrm{red}}^{I}(p) \hookrightarrow$ $\mathcal{M}_{A}^{I}(p)$ induces a homotopy equivalence on $K$-theory:

$$
K(\iota): K\left(\mathcal{M}_{A, \text { red }}^{I}(p)\right) \rightarrow K\left(\mathcal{M}_{A}^{I}(p)\right) .
$$

Proof. First assume that $p \geqslant n$. Then every $A$-module $M$ has $\operatorname{Projdim} M \leqslant n$, so $\mathcal{M}_{A}^{I}(p)=\mathcal{M}_{A}^{I}$ and $\mathcal{M}_{A, \text { red }}^{I}=\mathcal{M}_{A / I}$. In this case, the result was proven by Quillen in $[\mathbf{2 2}]$.

Next we assume that $n \geqslant p \geqslant \# S$. Then the inclusion functors

$$
\mathcal{M}_{A, ?}^{I}(p) \hookrightarrow \mathcal{M}_{A, ?}^{I}(n) \quad \text { and } \quad \mathcal{M}_{A, \text { red }}^{I}(k) \hookrightarrow \mathcal{M}_{A}^{I}(k) \quad(k=p, n)
$$

yield the commutative diagram below:

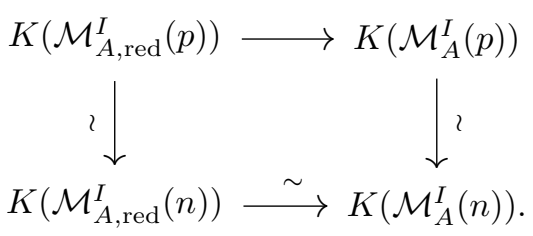

Here the vertical maps and the bottom horizontal map are homotopy equivalences by 5.8 and the first paragraph, respectively. Hence we obtain the result.

Recall the definition of $\mathcal{M}_{A, ?}\left(\mathfrak{f}_{U} ; \mathfrak{f}_{V}\right)(p)$ as a subcategory of $\mathbf{C u b}^{V}\left(\mathcal{M}_{A}^{\mathfrak{f}_{U}}\right)$ from 5.4.

Corollary 6.2. For any disjoint decomposition $S=U \sqcup V$, and integer $p \geqslant \# U$, the inclusion functor $\mathcal{M}_{A, \text { red }}\left(\mathfrak{f}_{U} ; \mathfrak{f}_{V}\right)(p) \hookrightarrow \mathcal{M}_{A}\left(\mathfrak{f}_{U} ; \mathfrak{f}_{V}\right)(p)$ induces a homotopy equivalence on $K$-theory:

$$
K\left(\mathcal{M}_{A, \text { red }}\left(\mathfrak{f}_{U} ; \mathfrak{f}_{V}\right)(p)\right) \rightarrow K\left(\mathcal{M}_{A}\left(\mathfrak{f}_{U} ; \mathfrak{f}_{V}\right)(p)\right)
$$

Proof. The inclusion functors $\mathcal{M}_{A, \text { red }}^{\mathfrak{f} U \sqcup T}(p+\# T) \hookrightarrow \mathcal{M}_{A}^{\mathfrak{f} U \sqcup T}(p+\# T)$ for any $T \in \mathcal{P}(V)$ and $\mathcal{M}_{A \text {,red }}\left(\mathfrak{f}_{U} ; \mathfrak{f}_{V}\right)(p) \hookrightarrow \mathcal{M}_{A}\left(\mathfrak{f}_{U} ; \mathfrak{f}_{V}\right)(p)$ and the exact functor $\lambda$ in 5.14 (2) yield the commutative diagram below:

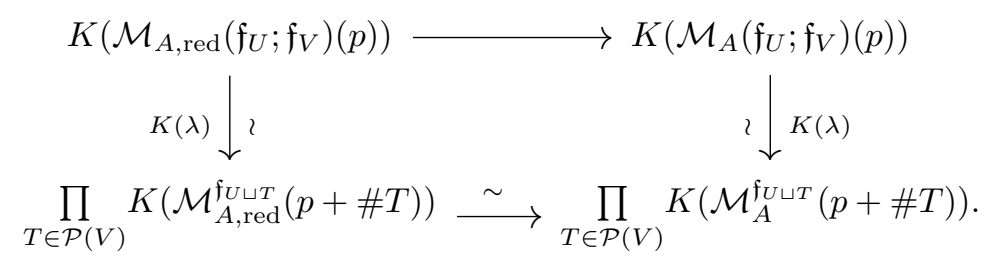

Here the vertical maps and the horizontal bottom map are homotopy equivalence by 5.14 (2) and 6.1, respectively. Hence we obtain the result.

Recall the definitions of $\mathbf{K}^{\mathfrak{f}_{A}}{ }_{A}^{\mathfrak{f}_{S}}$ from 4.8 and $\mathbf{K} \mathbf{s}_{A, \text { red }}^{\mathfrak{f}_{S}}$ and tq from 5.4. 
Corollary 6.3. The canonical inclusion functor $\iota$ : $\operatorname{Kos}_{A, \text { red }}^{\mathfrak{f}_{S}} \hookrightarrow \mathbf{K o s}_{A}^{\mathfrak{f}_{S}}$ induces the following homotopy equivalences on $K$-theory:

$$
\begin{aligned}
K\left(\mathbf{K o s}_{A, \text { red }}^{\mathfrak{f}_{S}}\right) & \rightarrow K\left(\mathbf{K o s}_{A}^{\mathfrak{f}_{S}}\right) \\
K\left(\mathbf{K o s}_{A, \text { red }}^{\mathfrak{f}_{S}} ; \mathrm{tq}\right) & \rightarrow K\left(\operatorname{Kos}_{A}^{\mathfrak{f}_{S}} ; \text { tq }\right) .
\end{aligned}
$$

Proof. If $S=\emptyset$, then $\operatorname{Kos}_{A}^{\mathfrak{f}_{S}}=\operatorname{Kos}_{A, \text { red }}^{\mathfrak{f}_{S}}=\mathcal{P}_{A}$. In this case, the assertion is trivial. We assume $\# S \geqslant 1$. Since we have the equality (7) in 5.5 (3), the first homotopy equivalence is just the special case $U=\emptyset, p=0$ of 6.2 . Let us consider the commutative diagram induced from the exact functor $\mathrm{H}_{0}$ Tot: $\left(\operatorname{Kos}_{A, ?}^{\mathfrak{f}_{S}}, \mathrm{tq}\right) \rightarrow \mathcal{M}_{A, ?}^{\mathfrak{f}_{S}}(\# S)$ defined in 5.14 (1) and the inclusion functors $\operatorname{Kos}_{A, \text { red }}^{\mathfrak{f}_{S}} \hookrightarrow \operatorname{Kos}_{A}^{\mathfrak{f}_{S}, ?}$ and $\mathcal{M}_{A, \text { red }}^{\mathfrak{f}_{S}}(\# S) \hookrightarrow$ $\mathcal{M}_{A}^{\mathfrak{f}_{S}}(\# S)$ below:

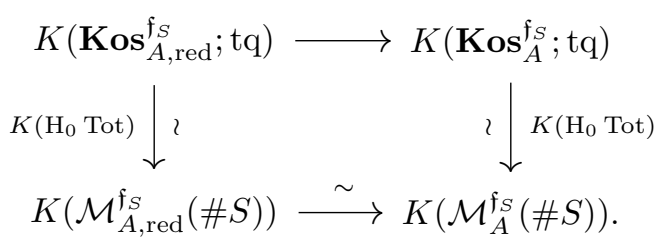

The vertical lines above are homotopy equivalences by 5.14 (1). The bottom horizontal line above is also a homotopy equivalence by 6.1 . Hence we obtain the second homotopy equivalence.

Proof of Corollary 0.5. By 5.10 and 5.11, we have the homotopy equivalences

$$
\underset{\lim _{S}}{\longrightarrow} K\left(\mathcal{M}_{A}^{\mathfrak{g}_{S}}(\# S)\right) \stackrel{\sim}{\rightarrow} K\left(\mathcal{M}_{A}^{\# S}(\# S)\right) \stackrel{\sim}{\rightarrow} K\left(\mathcal{M}_{A}^{\# S}\right),
$$

where $\mathfrak{g}_{S}$ runs over $A$-regular sequences indexed by $S$. Therefore Gersten's conjecture for $A$ is equivalent to the following assertion:

For any A-regular sequence $\left\{g_{s}\right\}_{s \in S}$ in $A$, the inclusion functor $\mathcal{M}_{A}^{\mathfrak{g}_{S}}(\# S) \hookrightarrow$ $\mathcal{M}_{A}^{\# S-1}$ induces the zero maps on $K$-groups.

Since $A$ is local, every $A$-regular sequence is an $A$-sequence by 4.1 (3). Fix an $A$ regular sequence $\mathfrak{f}_{S}=\left\{f_{s}\right\}_{s \in S}$ in $A$ and write $j$ for the inclusion functor $\mathcal{M}_{A}^{\mathfrak{f}_{S}}(\# S) \hookrightarrow$ $\mathcal{M}_{A}^{\# S-1}$. Then let us consider the commutative diagram below:

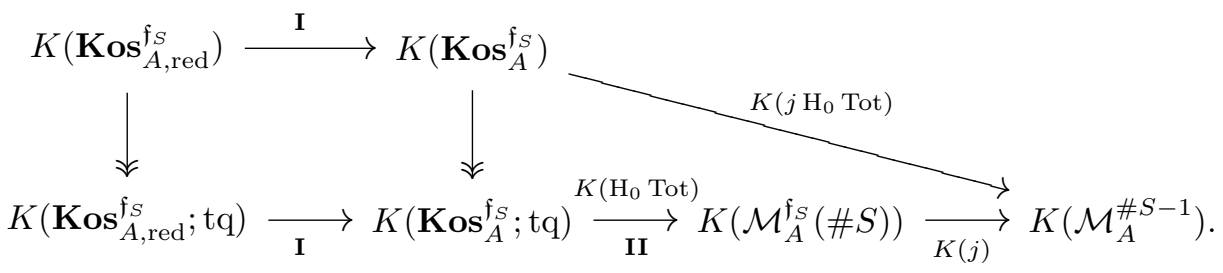

Here the maps I and II are homotopy equivalences by 6.3 and 5.14 (1), respectively, and the vertical maps are (split) epimorphisms by $5.14(3)$. Hence $K(j)$ is trivial if and only if the composition $K\left(j \mathrm{H}_{0}\right.$ Tot $): K\left(\operatorname{Kos}_{A, \text { red }}^{\mathfrak{f}_{S}}\right) \rightarrow K\left(\mathcal{M}_{A}^{\# S-1}\right)$ is trivial. Therefore we get the desired result. 


\section{Acknowledgments}

The author wishes to express his deep gratitude to Daniel R. Grayson, Kei Hagihara, Akiyoshi Sannai and Seidai Yasuda for stimulating discussions, Kei-ichi Watanabe for instructing him in the direct proof of 4.15 for the case of $\# S=2$ and Masanori Asakura, Toshiro Hiranouchi, Makoto Matsumoto, Nobuo Tsuzuki and Roozbeh Hazrat for inviting him to their universities. He would also like to thank the referees and Charles A. Weibel for carefully reading a preprint version of this paper and giving innumerable and valuable comments to make the paper more readable and Amnon Neeman for encouraging him in finishing the work.

\section{References}

[1] P. Balmer, Niveau spectral sequences on singular schemes and failure of generalized Gersten conjecture, Proc. Amer. Math. Soc. 137 (2009), 99-106.

[2] S. Bloch, A note on Gersten's conjecture in the mixed characteristic case, Contemp. Math. 55, Part I (1986), 75-78.

[3] N. Bourbaki, Éléments de mathématique. Algèbre commutative. Chapitre 10, Springer-Verlag, Berlin, 2007.

[4] D. A. Buchsbaum and D. Eisenbud, What makes a complex exact?, J. Algebra 25 (1973), 259-268.

[5] L. Claborn and R. Fossum, Generalizations of the notion of class group, Illinois J. Math. 12 (1968), 228-253.

[6] V. Diekert, Eine Bemerkung zu freien moduln über regulären lokalen ringen, J. Algebra 101 (1986), 188-189.

[7] S. P. Dutta, M. Hochster and J. E. McLaughlin, Modules of finite projective dimension with negative intersection multiplicities, Invent. Math. 79 (1985), 253-291.

[8] S. P. Dutta, A note on Chow groups and intersection multiplicity of modules, J. Algebra 161 (1993), 186-198.

[9] S. P. Dutta, On Chow groups and intersection multiplicity of modules, II, J. Algebra 171 (1995), 370-381.

[10] S. Gersten, Some exact sequences in the higher $K$-theory of rings, Lecture Notes in Math 341 (1973), 211-243.

[11] H. Gillet and M. Levine, The relative form of Gersten's conjecture over a discrete valuation ring: The smooth case, J. Pure Appl. Algebra 46 (1987), 59-71.

[12] H. Gillet and C. Soulé, Intersection theory using Adams operations, Invent. Math. 90 (1987), 243-277.

[13] D. R. Grayson, Exact sequences in algebraic K-theory, Illinois J. Math., 31 (1987), 598-617.

[14] D. R. Grayson, Adams operations on higher K-theory, K-Theory 6 (1992), $97-111$. 
[15] T. Hiranouchi and S. Mochizuki, Pure weight perfect modules over divisorial schemes, Aspects Math. E40 (2010), 75-89.

[16] B. Keller, Chain complexes and stable categories, Manuscripta Math. 67 (1990), 379-417.

[17] M. Levine, A K-theoretic approach to multiplicities, Math. Ann. 271 (1985), 451-458.

[18] M. Levine, Localization on singular varieties, Invent. Math. 91 (1988), 423-464.

[19] H. Matsumura, Commutative ring theory, Cambridge University Press, Cambridge, 1986.

[20] S. Mochizuki, Higher algebraic $K$-theory of finitely generated torsion modules over principal ideal domains, available at http://www.math.uiuc.edu/ K-theory/0823/ (2007).

[21] I. Panin, The equi-characteristic case of the Gersten conjecture, Proc. Steklov Inst. Math. 241 (2003), 154-163.

[22] D. Quillen, Higher algebraic K-theory I, Lecture Notes in Math. 341 (1973), $85-147$.

[23] G. Segal, Categories and cohomology theories, Topology 13 (1974), 293-312.

[24] J. P. Serre, Algèbre locale, Springer-Verlag, Berlin-New York, 1965.

[25] W. Smoke, Perfect Modules over Cohen-Macaulay local rings, J. Algebra 106 (1987), 367-375.

[26] R. E. Staffeldt, On fundamental theorems of algebraic $K$-theory, $K$-Theory 2 (1989), 511-532.

[27] R. W. Thomason and T. Trobaugh, Higher algebraic $K$-theory of schemes and of derived categories, Progr. Math., vol. 88 (1990), 247-435.

[28] F. Waldhausen, Algebraic K-theory of generalized free products, Ann. of Math. (2) 108 (1978), 135-256.

[29] F. Waldhausen, Algebraic K-theory of spaces, Lecture Notes in Math. 1126 (1985), 318-419.

[30] C. A. Weibel, An introduction to homological algebra, Cambridge University Press, Cambridge, 1994.

[31] C. A. Weibel, The K-book. An introduction to algebraic K-theory, American Mathematical Society, Providence, RI, 2013.

Satoshi Mochizuki mochi@gug.math.chuo-u.ac.jp

Department of Mathematics, Chuo University, Bunkyoku, Tokyo, Japan 